\title{
Further Examination of the Mechanism of Round Synthetic Jets in Delaying Turbulent Flow Separation
}

\author{
Shan Zhong • Shanying Zhang
}

Received: 30 September 2012 / Accepted: 16 May 2013 / Published online: 7 June 2013

(C) Springer Science+Business Media Dordrecht 2013

\begin{abstract}
This paper reports the findings from a further study of the 2D and stereo PIV data obtained in the interaction zone between the separated turbulent boundary layer over a 2D ramp and round synthetic jets by the authors. The synthetic jets are operated at two actuation frequencies with one being close to the natural frequency of the separated shear layer. Both the triple decomposition technique and Qcriterion are employed to investigate how the separated flow responds to the passage of different parts of the vortical structures produced by the synthetic jets during an actuation cycle at different synthetic jet operating conditions. An attempt is made to explain the observed differences in the ways that the separated flow responds to the actuation of synthetic jets at the two actuation frequencies. A better understanding of the mechanism of flow separation delay using round synthetic jets is obtained, leading to a more complete physical model describing the interaction mechanism.
\end{abstract}

Keywords Synthetic jets · Flow separation control · Turbulent boundary layer

\section{Nomenclature}

D diameter of cavity or orifice, $\mathrm{mm}$

f diaphragm oscillation frequency, $\mathrm{Hz}$

h ramp height, $\mathrm{mm}$

$\mathrm{L}_{0} \quad$ stroke length, $L_{0}=\bar{U}_{o} T$

L dimensionless stroke length, $L=\frac{L_{o}}{D_{o}}$

$\mathrm{Re}_{\mathrm{L}} \quad$ Reynolds number based on dimensionless stroke length, $R e_{L}=\frac{\bar{U}_{o} L_{o}}{v}$

$\mathrm{St}_{\mathrm{h}} \quad$ Strouhal number, $S t_{h}=\frac{f h}{U_{\infty}}$

Shan Zhong $(\varangle) \cdot$ Shanying Zhang

School of Mechanical, Aerospace and Civil Engineering,

Manchester University, Manchester, UK

e-mail: shan.zhong@manchester.ac.uk 


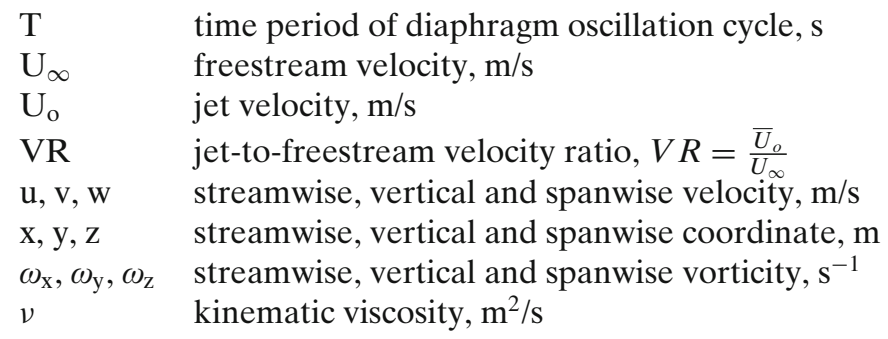

Superscripts and other notations

$\begin{array}{ll}- & \text { time-averaged values } \\ <> & \text { phase-averaged values } \\ \sim & \text { periodic values } \\ , & \text { random values }\end{array}$

\section{Introduction}

Interest in active flow separation control in conditions akin to those on aircraft components operating in high-load conditions has stimulated the development of actuators that produce localized disturbances in a flow field since 1990s [9]. Among them, synthetic jet actuators, which create pressure fluctuations with an oscillating diaphragm in a cavity and eject a net vorticity through an orifice opening into the flow with a zero net mass flux, have received particular attention (e.g. [2, 14, 23, 24, $29,33,39,41])$. Extensive review of the existing research on synthetic jets can be found in Glezer and Amitay [12] and Cattafesta and Sheplak [5].

The research on synthetic jets can be classified based on the orifice geometry. (see [29]), i.e. plane 2-D or very-high-aspect-ratio slots (with aspect ratio of 75 or above), circular, elliptic or low-aspect-ratio slots (with aspect ratio below 5) and rectangular or finite-span slots (with aspect ratio between 5 and 75). So far, the research on synthetic jets reported in the literature has been dominated by $2 \mathrm{D}$ slot jets, especially with respect of separation control (e.g. [2,11,25]). This is because the nominal twodimensional nature of a slot synthetic jet, which spans the entire spanwise extent of the flow, simplifies both measurements and numerical simulations [21]. More recently, the behaviour of finite-span synthetic jets also has received research attention, exemplified by the work of Amitay and Cannelle [1], Sahni et al. [29].

The work reported in this paper focuses on round synthetic jets due to their simple orifice geometry and yet resultant complex three-dimensional flow structures. A round synthetic jet ejecting into a quiescent fluid typically produces a train of vortex rings, which interact with each other and decay, as they propagate away from the jet orifice at their own self-induced velocities $[4,12,14]$. When issuing into a boundary layer, these vortex rings are subjected to a combined impact of the shear and the vorticity in the boundary layer as well as the Magnus force [6] produced by the crossflow (see $[16,31,42])$. Hence they will undergo certain degrees of tilting and deformation 
depending on their strength and resident time within the boundary layer, resulting in the formation of complex three-dimensional vortical structures. The dye visualisation experiment of a single round synthetic jet issuing into a flat plate laminar boundary layer carried out by Jabbal and Zhong [18] revealed that, at a given actuation frequency, as the jet-to-freestream-velocity ratio increases, the primary vortical structures produced by such an interaction, first appear as hairpin-like vortices that are located close to the wall, then as tilted vortex rings with a pair of trailing legs that penetrate the edge of the boundary layer shortly downstream. Similar flow structures were also reproduced in the LES simulations by Sau and Mahesh [30], although their vortex rings were created without a suction cycle.

Despite the elevated level of shear and turbulent diffusion present in a turbulent boundary layer, similar hairpin type of vortical structures also has been observed by Zaman and Milanovic [37] and Garcillan et al. [10] in a flat plate turbulent boundary layer at moderate jet-freestream velocity ratios. Numerical simulations conducted in a turbulent boundary layer reveal that these primary structures also induce a further pair of streamwise vortices underneath themselves, which plays a key role in entraining high-momentum fluids towards the near-wall region [36]. These findings tie in well with the oil flow patterns observed by Crook et al. [7] in a turbulent flow over a circular cylinder. They are also confirmed by Zhang and Zhong [40], who studied the interaction between an incipiently separated turbulent boundary layer over a 2D ramp and round synthetic jets and thereby established a conceptual model of the interaction mechanism. However, whether the primary vortical structures, such as the hairpin vortices, play a direct role in delaying flow separation is still unclear. Yet such information is essential for building a more complete physical model for flow separation delay, which involves a hierarchy of vortical structures.

A periodic actuation of synthetic jets is expected to promote mixing desirable for flow separation delay via formation of time-dependent coherent vortices and production of additional random turbulence. In the case of slot synthetic jets, a significant enhancement in flow separation delay can also be achieved by tuning the actuation frequency towards the $2 \mathrm{D}$ instability modes as shown in the experiments of Greenblatt et al. [11], and the numerical simulations of Dandois et al [8] and Kotapati et al. [20]. An optimal frequency for flow control was reported to exist around the shear layer frequency or shedding frequency of the separated flow. The shear layer mode is linked to the Kelvin-Helmholtz instabilities in the separated shear layer. Hasan and Khan [13] showed that this mode is characterised by the Strouhal number $\mathrm{St}_{\theta}=0.011$, where $\theta$ is the momentum thickness at the location of separation. The shedding mode, however, is independent from the state of the shear layer and it characterised by $\mathrm{St}_{\mathrm{h}}=0.185$, where $\mathrm{h}$ is the height of the ramp which creates the flow separation. It is shown that actuating a slot synthetic jet at the above frequencies encourages the formation of large-scale spanwise roller vortices, which entrain high-momentum fluids to the near-wall region resulting in a strong delay of flow separation [11]. Due to the confined spanwise extent of round synthetic jets, however, a separated shear layer is expected to respond differently to the actuation of round synthetic jets. In view of the very limited amount of research reported in the literature on the interaction of round synthetic jets with a separated boundary layer, either experimentally or numerically, further investigations are still required in order to obtain a better understanding of the associated flow physics. 
In the recent experiment carried out by Zhang and Zhong [40], 2D PIV and stereo PIV techniques were employed to provide detailed information about the flow field in the interaction zone between an incipiently separated boundary layer over a 2D ramp and round synthetic jets at $\mathrm{VR}=0.2$ to 0.5 at two actuation frequencies $\left(\mathrm{St}_{\mathrm{h}}=\right.$ 0.2 and 0.6). It was found that at $\mathrm{St}_{\mathrm{h}}=0.2$ the top boundary of reverse flow fluctuates and the separated shear layer exhibits a flapping motion during the actuation cycle. At $\mathrm{St}_{\mathrm{h}}=0.6$, however, the height of reverse flow remains suppressed throughout the entire actuation cycle and the flapping of shear layer is not evident. Furthermore, at $\mathrm{St}_{\mathrm{h}}=0.2$ there is a profound reduction in the time-averaged height of reverse flow at a velocity ratio of $\mathrm{VR}=0.2$, whereas the improvement becomes less significant with a further increase in velocity ratio. In contrast, at $\mathrm{St}_{\mathrm{h}}=0.6$ although the extent in the flow separation delay is less profound at $\mathrm{VR}=0.2$, it increases progressively with increasing velocity ratios. Although the differences in the response of the separated flow to an increasing velocity ratio at $\mathrm{St}_{\mathrm{h}}=0.2$ and 0.6 are related to the fact that $\mathrm{St}_{\mathrm{h}}=0.2$ is close to the shear layer frequency, the detailed interaction mechanism between the separated flow and the synthetic jets is still unclear.

In this paper, the PIV datasets obtained by Zhang and Zhong [40] are further explored along with new power spectra deduced from a LDA measurement. In particular, the triple decomposition technique and Q-criterion are employed to investigate the interaction between the separated flow and round synthetic jets, leading to new findings which have not been reported earlier. The specific objectives of this paper are threefold: a). to investigate how the separated flow responds to the passage of the vortical structures produced by the synthetic jets in terms of periodic flow structures and turbulence structures; b). to examine the role of both the hairpin vortices and the streamwise vortices produced by the synthetic jet in delaying flow separation; c). to identify the reasons for the different response of the separated flow to an increasing velocity ratio at $\mathrm{St}_{\mathrm{h}}=0.2$ and 0.6 . Incorporating the new findings from this study, a more complete physical model describing the mechanism by which round synthetic jets interact with an incipiently separated boundary layer is presented. The knowledge gained from this study will be useful for deriving costeffective operational flow-control solutions for practical settings.

\section{Experimental Setup, Measurement Techniques and Methods of Data Reduction}

\subsection{The experimental setup}

The experiments are conducted in the boundary layer tunnel at the School of Mechanical, Aerospace and Civil Engineering at Manchester University. It is a blowdown wind tunnel and has a test section which is $1.2 \mathrm{~m}$ wide, $0.3 \mathrm{~m}$ high and $5.49 \mathrm{~m}$ in length. A trip wire of $2 \mathrm{~mm}$ in diameter is fixed circumferentially at $0.5 \mathrm{~m}$ upstream of the inlet of the test section to ensure the formation of turbulent boundary layers along the walls of the test section. Measurements have shown that the boundary layers developing along the top and bottom floor are two-dimensional across at least $70 \%$ of the span.

In the present experiments, a $2 \mathrm{D}$ ramp with a height of $\mathrm{h}=31.5 \mathrm{~mm}$ with a similar design as that used by Song and Eaton [32] is used to produce a separated turbulent boundary layer to which flow control using synthetic jets is applied. It is mounted on the ceiling of the test section at a distance of $800 \mathrm{~mm}$ downstream of its inlet as 
shown in Fig. 1a. For the precise geometry of the 2D ramp, please refer to the paper by Bentaleb et al. [3] in which the mathematical formulae for its shape are given.

The synthetic jet array consists of three jets, which are aligned normal to the freestream direction and located at $20 \mathrm{~mm}$ upstream of the start of the ramp-down section (see Fig. 1b). The jet orifices have a diameter of $\mathrm{D}_{\mathrm{o}}=5 \mathrm{~mm}$ and are spaced $50 \mathrm{~mm}\left(10 \mathrm{D}_{\mathrm{o}}\right)$ apart. For the jets to be more effective, in this experiment the streamwise distance between the array and the onset of separation is chosen to be the smallest distance that is possible in the current setup, which is $55 \mathrm{~mm}$ or $12 \mathrm{D}_{\mathrm{o}}$. The coordinate system used in this paper is specified in Fig. 1. The origin of the coordinate system is located on the test surface at the start of the ramp-down section along the central plane of the middle jet. Three synthetic jet actuators are used to create these three jets. Although each actuator has its own separate cavity, the movement of their diaphragms is driven by a single mechanical mechanism. More detailed information about the synthetic jet actuators can be found in Zhang and Zhong [40].

\subsection{Laser Doppler Anemometry (LDA)}

A DANTEC three-component LDA system is employed in the experiment. It is firstly used to measure the velocity profiles across the boundary layer as well as to determine the precise locations of flow separation and reattachment over the ramp-down section for the baseline flow without jet actuation. It is then used to measure two velocity components simultaneously across the boundary layer in both the baseline case and the controlled cases from which the energy spectra are deduced.

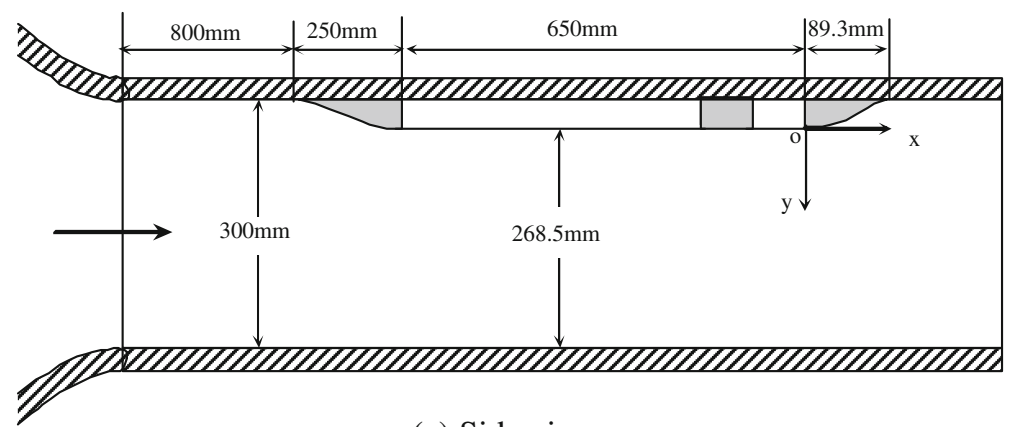

(a) Side view

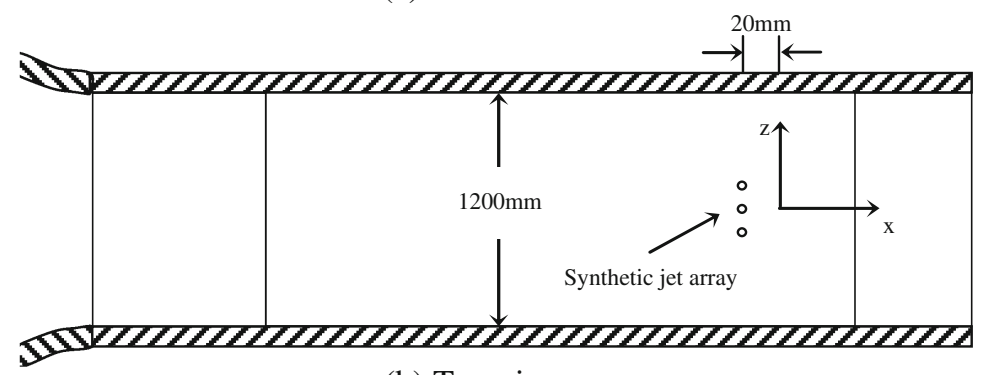

(b) Top view

Fig. 1 The geometry of the tunnel test section and the 2D ramp (not to scale) 
The measurement volume of the LDA system has an ellipsoid shape, which has a minor axial length of $0.05 \mathrm{~mm}$ and a major axial length of $1.2 \mathrm{~mm}$, and its major axis is aligned in the spanwise direction of the tunnel test section. A computer controlled 3D traverse mechanism is used to provide an accurate positioning of the measurement volume with a resolution of $6.25 \mu \mathrm{m}$ in the three orthogonal directions. The data rate typically varies from 200 to 2000 samples/sec from the near-wall region to the freestream. To obtain reliable time-averaged velocity and turbulence information, the sample size is set at $10^{4}$ at each measurement point.

Since LDA signals consist of a data series with uneven time intervals, the 'Resample and Hold' technique described by Nobach [26] is firstly used to construct the equivalent data series with an equally-spaced time interval required for power spectrum analyses. The latter data series is then divided into a number of data blocks of an adequate length depending on the range and resolution of frequency required. Finally, the power spectrum of the data series is obtained by taking the average of the power spectra of all the data blocks.

\subsection{D Particle Image Velocimetry (PIV) and stereo PIV}

In the present experiments, both 2D PIV and stereo PIV measurements are undertaken to capture the three-dimensional interaction between the synthetic jets and the separated flow over the ramp-down section. The details about the PIV setup can be found in Zhang and Zhong [40] and will not be repeated here.

The 2D PIV data are obtained on several streamwise planes on one side of the middle jet with a spanwise spacing of $5 \mathrm{~mm}$ using a 4-Mega pixel PIV camera. The flow is assumed to be symmetric relative to the central plane of the middle jet for the purpose of flow field reconstruction. For the controlled cases, the images are taken at eight equally-spaced phases in a synthetic jet actuation cycle and the phaseaveraged velocity field is obtained by averaging 200 phase-locked measurements. The stereo PIV measurements are conducted on three cross-stream planes downstream of the middle jet over the ramp-down section at $\mathrm{x} / \mathrm{h}=1,2$ and 2.5. PIV images are taken at eight equally spaced phases in a synthetic jet actuation cycle and the phase-averaged velocity field is obtained by averaging 250 phase-locked measurements.

In the present setup, the uncertainty in the camera domain displacement is about 0.2 pixels, which results in an uncertainty of $2 \%$ in the velocity measurements with a pulse time separation of $80 \mu \mathrm{s}$ at a freestream velocity of $6.5 \mathrm{~m} / \mathrm{s}$ used in the present experiment. The uncertainty of the phase-averaged turbulent shear stress deduced from the 2D PIV data is estimated by taking the rms value of its instantaneous fluctuations and it is found to be about $10 \%$ of the maximum turbulent shear stress in the separated shear layer with a $95 \%$ confidence interval. For the stereo PIV, the perspective error is removed and the in-plane error is reduced by a factor of $1 / \sqrt{2}$ whereas the out-of-plane error is increased by a factor of 2.8 relative to the in-plane error in the 2D PIV [27, 38]. Given the in-plane error in our 2D PIV measurement of $2 \%$, the measurement uncertainty in the in-plane and the out-ofplane velocity components in the 3D PIV is hence estimated to be about $1.5 \%$ and $4.2 \%$, respectively. 
2.4 Methods of data reduction

In the present study, in addition to the conventional time-averaging and phaseaveraging techniques, the triple decomposition technique [28] is used to analyse the characteristics of the flow field. In triple decomposition, a time series $f(x, y, z, t)$ measured at the location $(x, y, z)$ and time $t$ can be represent by

$$
f(x, y, z, t)=\bar{f}(x, y, z)+\tilde{f}(x, y, z, t)+f^{\prime}(x, y, z, t)
$$

where $\bar{f}(x, y, z)$ is the overall time-averaged signal, $\tilde{f}(x, y, z, t)$ is the periodic component and $f^{\prime}(x, y, z, t)$ is the random turbulent component. In practice, time averaging is first performed on the instantaneous velocity components, i.e. $u$ and $v$, to obtain the time-averaged values, $\bar{u}(x, y, z))$ and $\bar{v}(x, y, z))$. The phase-averaged velocity components, $\langle u(x, y, z, t)\rangle$ and $\langle v(x, y, z, t)\rangle$, are then obtained by averaging a sufficient number of measurements acquired at each phase of the synthetic jet actuation cycle. With the phase-averaged velocity components available, the instantaneous periodic and random velocity fluctuations can then be obtained from

$$
\begin{aligned}
& \widetilde{u}(x, y, z, t)=\langle u(x, y, z, t)\rangle-\bar{u}(x, y, z) \\
& \widetilde{v}(x, y, z, t)=\langle v(x, y, z, t)\rangle-\bar{v}(x, y, z)
\end{aligned}
$$

and

$$
\begin{aligned}
& u^{\prime}(x, y, z, t)=u(x, y, z, t)-\langle u(x, y, z, t)\rangle \\
& v^{\prime}(x, y, z, t)=v(x, y, z, t)-\langle v(x, y, z, t)\rangle
\end{aligned}
$$

From the above data, the time-averaged periodic and random Reynolds stresses $\overline{\tilde{u} \tilde{v}}$ and $\overline{u^{\prime} v^{\prime}}$ are calculated. Similarly, their phase-averaged counterparts $\langle\tilde{u} \widetilde{v}\rangle$ and $\left\langle u^{\prime} v^{\prime}\right\rangle$ are also computed. As such, the contributions of periodic motions and random turbulent structures to the flow separation delay can be evaluated separately.

Given the three velocity components in a three-dimensional space, the physical appearance of vortical structures can be reconstructed from the stereo PIV data using the $\mathrm{Q}$-criterion. The $\mathrm{Q}$-criterion, denoted as $Q$, is defined as the second invariant of the velocity gradient tensor, given for incompressible flow by

$$
Q=0.5\left(\Omega_{\mathrm{ij}} \Omega_{\mathrm{ij}}-\mathrm{S}_{\mathrm{ij}} \mathrm{S}_{\mathrm{ij}}\right)
$$

where $\mathrm{S}_{\mathrm{ij}}$ and $\Omega_{\mathrm{ij}}$ are symmetric and anti-symmetric parts of the velocity gradient tensor. A positive $Q$ presents the necessary condition for the existence of convex low-pressure tubes, which are generally associated with coherent vortices [15]. Hence the iso-surfaces of $Q(>0)$, which locate the regions where the strength of rotation overcomes that of the strain, depict the appearance of coherent vortices in the flow field.

In the present study, phase-averaged velocity fields in a synthetic jet actuation are only obtained with the stereo PIV at several cross-stream planes. However, by using the Taylor's frozen-flow hypothesis [35], the spatial extent of the coherent structures 
can be deduced from the temporal variations of flow field measured at a cross-stream plane when the convection velocity of these structures is known.

\section{Test Conditions}

\subsection{The baseline flow}

All the tests in this study are performed at a fixed freestream velocity of $U_{\infty}=6.5 \mathrm{~m} / \mathrm{s}$ and the freestream turbulent intensity is about $0.3 \%$. The distributions of both normalised velocity and velocity fluctuation profiles obtained using the LDA confirm that, after an initial acceleration at the ramp-on section, the boundary layer flow has reached an equilibrium condition at which mean-flow self-preservation prevails [34] at the location of the synthetic jet array.

Figure 2 a shows the contours of the time-averaged streamwise velocity component obtained using PIV on the central streamwise plane over the ramp-down section in

Fig. 2 Characteristics of the baseline flow; a contours of streamwise velocity; b contours of spanwise vorticity; c Streamline patterns showing the approximate extent of the separation bubble; d Power spectrum at $\mathrm{x} / \mathrm{h}=1.91$ and $\mathrm{y} / \mathrm{h}=-0.33$ in the separated shear layer
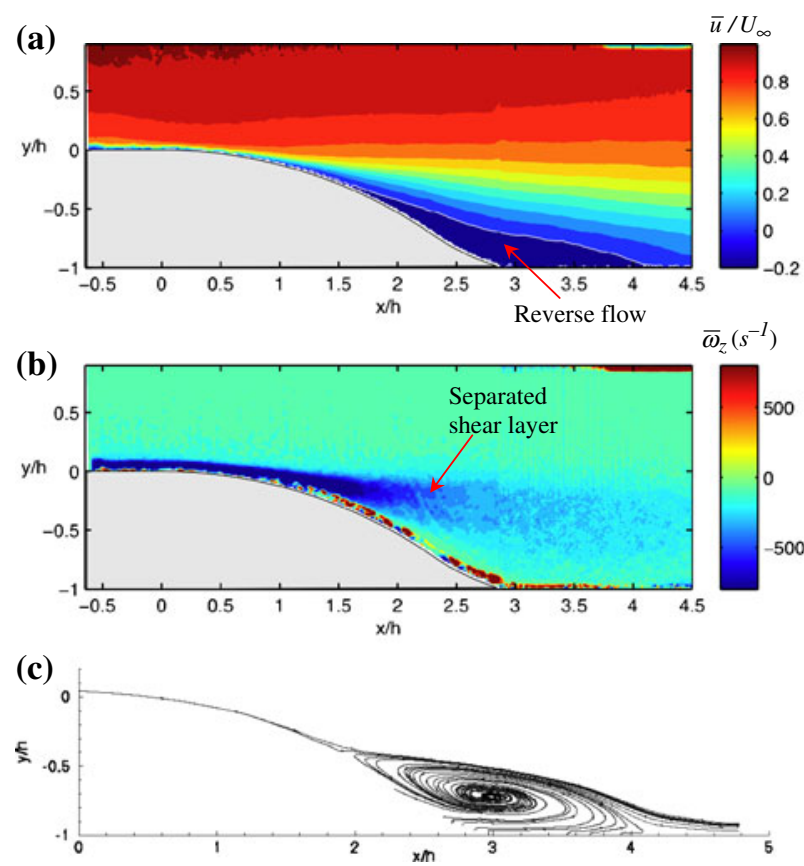

(d)

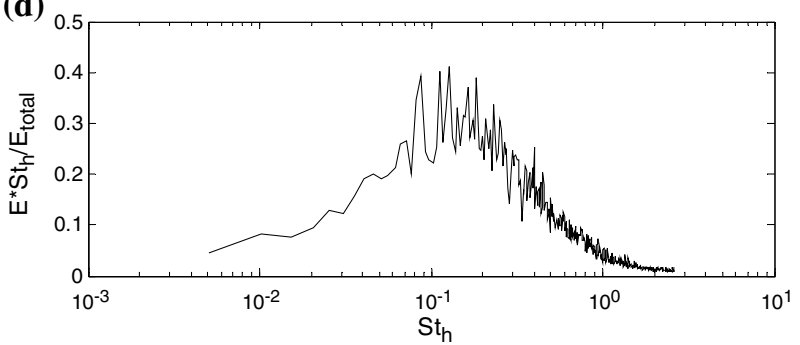


the baseline case. (The slight discontinuity in the contours at $\mathrm{x} / \mathrm{h}=2.85$ is caused by patching the PIV data obtained separately from the upstream and the downstream measurement area.) The top edge of the isolated reverse flow region is indicated by the white line. The separated shear layer can be seen as a region with a high level of negative spanwise vorticity (Fig. 2b). The approximate shape of the separation bubble can be visualised using streamlines as shown in Fig. 2c.

The exact locations of the separation and reattachment are deduced from the time-averaged streamwise velocity component measured using LDA at a very small distance $(0.05 \mathrm{~mm})$ from the wall along the centreline of the ramp-down section with fine streamwise steps. The locations of the separation and reattachment point are found to be located at $x=34.4 \mathrm{~mm}(\mathrm{x} / \mathrm{h}=1.1)$ and $x=132.7 \mathrm{~mm}(\mathrm{x} / \mathrm{h}=4.2)$ respectively. At the location of the synthetic jet array, the boundary layer thickness is $26.8 \mathrm{~mm}$ and the Reynolds number based on the local momentum thickness is 984. More measurement results can be found in Zhang and Zhong [40]. A largeeddy simulation undertaken on the same ramp flow, which offers an insight into the physics of flow separation and a valuable data set for benchmarking model solutions and investigating statistical turbulence-closure proposals, is described in Bentaleb et al. [3].

\subsection{Operating conditions of synthetic jet actuators}

In the present experiment, both the spacing between adjacent orifices in the synthetic jet array and the distance between the array and the start of the ramp-down are fixed. Based on dimensional analysis $[18,41]$, the behaviour of synthetic jets issuing into a given boundary layer is determined by the dimensionless stroke length $L$, the Reynolds number defined based on the stroke length $R e_{\mathrm{L}}$, the velocity ratio $V R$ and the dimensionless frequency $S t_{\mathrm{h}}$.

The definitions and a brief discussion of the physical meaning of $L, R e_{L}$ and VR can be found in Zhong et al. [41] and Jabbal and Zhong [18]. Here it is emphasised that VR is defined using the time-averaged blowing velocity over the entire cycle of the synthetic jet, $\bar{U}_{o}$. Assuming incompressible flow in each actuator, it can be shown that the time-averaged blowing velocity during the entire cycle at the orifice exit for the present actuator is expressed as

$$
\bar{U}_{o}=f \Delta\left(\frac{D_{c}}{D_{o}}\right)^{2}
$$

and the peak velocity is equal to $\pi \bar{U}_{o}$. Here $\mathrm{D}_{\mathrm{c}}$ is the diameter of the cavity, $f$ and $\Delta$ are the diaphragm oscillating frequency and peak-to-peak displacement respectively. Since Eq. 5 yields a predicted jet velocity which is in a good agreement with the measured velocity $[17,19]$, in this paper it is used to calculate the dimensionless parameters, which characterise the behaviour of synthetic jets. The Strouhal number is defined based on the freestream velocity and the ramp height. Hence $1 / S t_{\mathrm{h}}$ can be taken as an approximate measure of the distance between the consecutive structures produced by the synthetic jets as a fraction of the ramp height.

Detailed flow measurements are subsequently undertaken at VR $=0.2,0.3$ and 0.5 at two actuation frequencies, i.e. $f=40$ and $120 \mathrm{~Hz}$, corresponding to $\mathrm{St}_{\mathrm{h}}=0.2$ and 0.6 respectively. (The values of $L$ and $R e_{L}$ for these cases are given in Table 1.) An amplitude sweep has been carried out at $L=2$ and the results confirm that in 
Table 1 Values of $L$ and $R e_{L}$ for the test cases

\begin{tabular}{|c|c|c|c|c|c|c|}
\hline \multirow[t]{2}{*}{$S t_{\mathrm{h}}$} & \multicolumn{2}{|c|}{$\mathrm{VR}=0.2$} & \multicolumn{2}{|c|}{$\mathrm{VR}=0.3$} & \multicolumn{2}{|c|}{$\mathrm{VR}=0.5$} \\
\hline & $\mathrm{L}$ & $\operatorname{Re}_{\mathrm{L}}$ & $\mathrm{L}$ & $\operatorname{Re}_{\mathrm{L}}$ & $\mathrm{L}$ & $\operatorname{Re}_{\mathrm{L}}$ \\
\hline 0.2 & 6.5 & 14289 & 9.8 & 21434 & 16.3 & 35723 \\
\hline 0.6 & 2.2 & 4763 & 3.3 & 7145 & 5.4 & 11908 \\
\hline
\end{tabular}

the present experiment the actuators are operated at a range of frequencies, which are much lower than their resonance frequencies. Here the choice of $f=120 \mathrm{~Hz}$ is determined by the maximum capacity of the shaker which drives the actuators, whereas the choice of $f=40 \mathrm{~Hz}$ is to ensure that the actuators operate near the natural frequency of the separated shear layer. At $f=40 \mathrm{~Hz}$, the Strouhal numbers based on the momentum thickness at the point of separation in the baseline case and the ramp height are 0.011 and 0.2 respectively. They are very close to the correlations for the shear layer frequency $\left(\mathrm{St}_{\theta}=0.011\right)$ and the shedding frequency $\left(\mathrm{St}_{\mathrm{h}}=0.185\right)$ given by Hasan and Khan [13]. The presence of a peak around $\mathrm{St}_{\mathrm{h}}=0.2$ in the power spectrum obtained in the region of the separated shear layer also confirms that $\mathrm{f}=$ $40 \mathrm{~Hz}$ is indeed close to the natural frequency of the shear layer (see Fig. 2d). For this setup, the estimated shear layer mode and shedding mode are associated with almost

Fig. 3 Contours of phase-averaged streamwise velocity at $\mathrm{St}_{\mathrm{h}}=0.2$ and $\mathrm{VR}=$ 0.3 at four phases in the actuation cycle; $\mathbf{a} 0^{\circ}, \mathbf{b} 90^{\circ}$, c $180^{\circ}$ and $\mathbf{d} 270^{\circ}$. (The white line indicates the edge of the reverse flow in the baseline flow.)

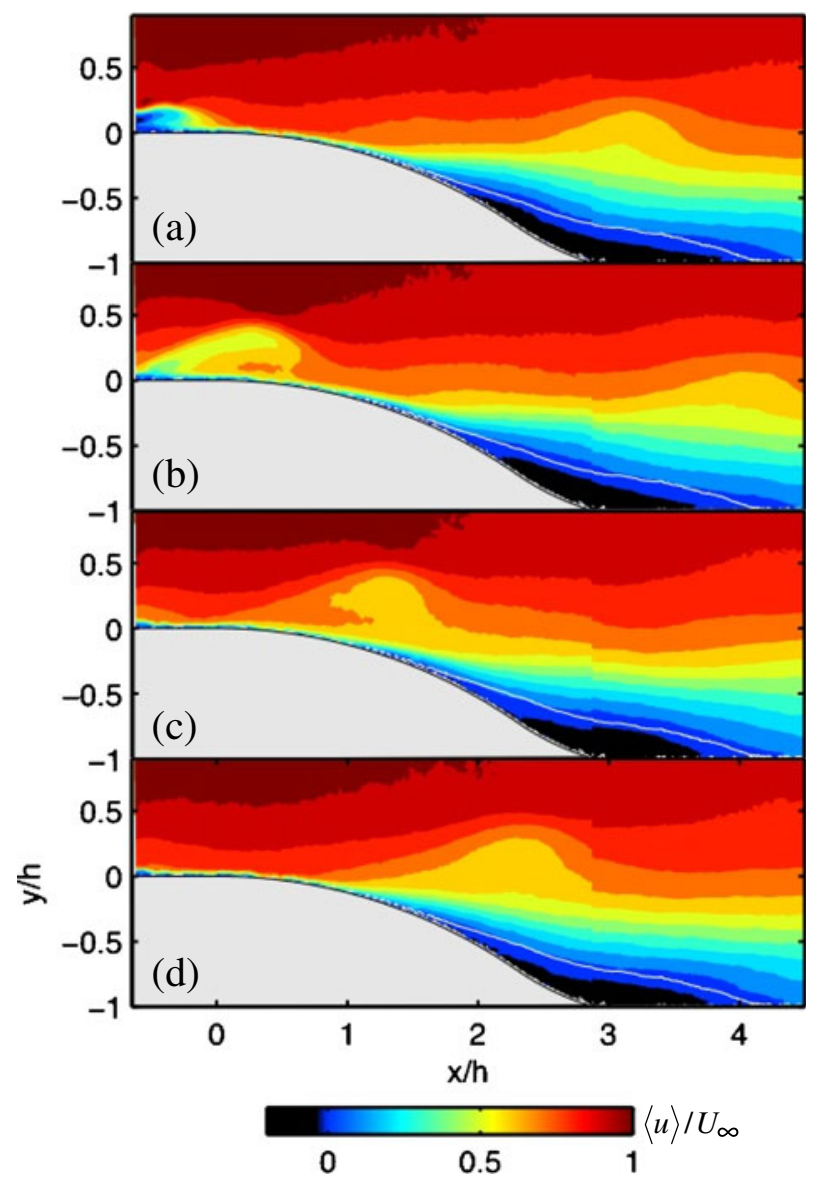


the same frequency due to the specific nature of the separated flow studied here, in which the momentum thickness of the boundary layer at the point of separation is about $5 \%$ of the ramp height. If the ramp height is much larger than the boundary layer thickness, the frequency of the shedding mode is expected to be lower than that of shear layer mode as a result of vortex pairing of the initial shear layer rollup.

\section{Results and Discussion}

A comprehensive LDA and PIV data set has been produced from the experiment, providing detailed information about the three-dimensional effect of the synthetic jets on the separated boundary layer.

\subsection{Temporal variations in the flow control effect}

Both the time-averaged control effect and temporal variations in the control effect of the synthetic jets have been discussed in Zhang and Zhong [40]. In this paper,

Fig. 4 Contours of phase-averaged spanwise vorticity $\left(\omega_{\mathrm{z}}\right)$ with superimposed velocity vectors at $\mathrm{St}_{\mathrm{h}}=0.2$ and $\mathrm{VR}=0.3$ at four phases in the actuation cycle; a $0^{\circ}$, b $90^{\circ}$, c $180^{\circ}$ and d $270^{\circ}$ phase of the actuation cycle

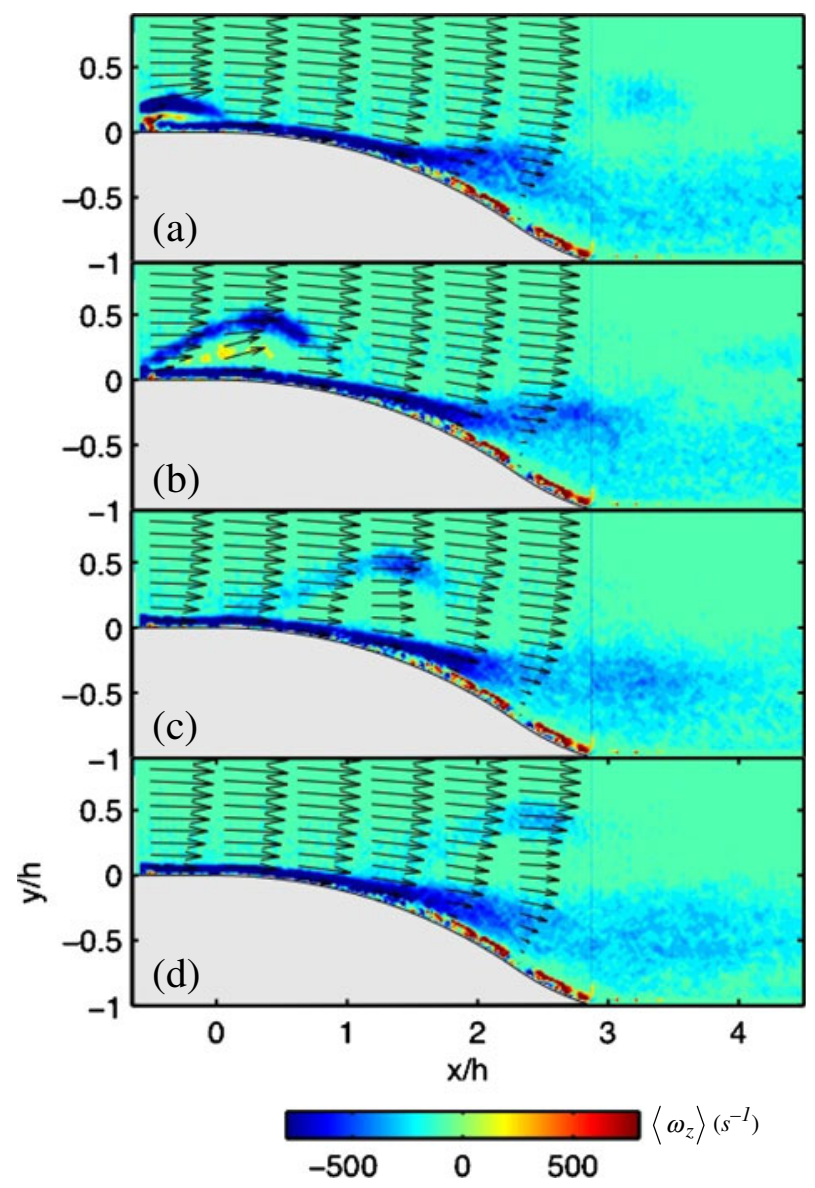


the contours of phase-averaged streamwise velocity at $\mathrm{VR}=0.3$ are illustrated to aid the understanding of the periodic and turbulent structures presented later in this paper. Note that these contours are not a duplication of those presented in Zhang and Zhong [40] due to the use of a different VR.

The contours of normalised phase-averaged streamwise velocity $\langle u\rangle / U_{\text {ref }}$ on the streamwise central plane of the middle jet at $\mathrm{St}_{\mathrm{h}}=0.2$ and $\mathrm{VR}=0.3$ at four different phases in a synthetic jet actuation cycle are shown in Fig. 3. In these contours, the low-speed bulges caused by an up-wash produced by the synthetic jets appear as the dominant features. The top boundary of the reverse flow region moves downwards just before the arrival of the low-speed budge, and it bounces back as this structure has passed. Figure 4 reveals that at $\mathrm{St}_{\mathrm{h}}=0.2$ the aforementioned low-speed bulge is associated with a strong negative spanwise vorticity, which is of the same sign as that in the boundary layer. The separated shear layer, which is associated with a high level of negative spanwise vorticity, appears like a ribbon, exhibiting a flapping motion during the synthetic jet actuation cycle; it is deflected downwards locally as the aforementioned vortical structure arrives (Fig. 4c) and bounces back as the vortical structure has gone past (Fig. 4d).

Fig. 5 Contours of phase-averaged streamwise velocity at $\mathrm{St}_{\mathrm{h}}=0.6$ and $\mathrm{VR}=$ 0.3 at four phases in the actuation cycle; $\mathbf{a} 0^{\circ}, \mathbf{b} 90^{\circ}$, c $180^{\circ}$ and $\mathbf{d} 270^{\circ}$. (The white line indicates the edge of the reverse flow in the baseline flow)

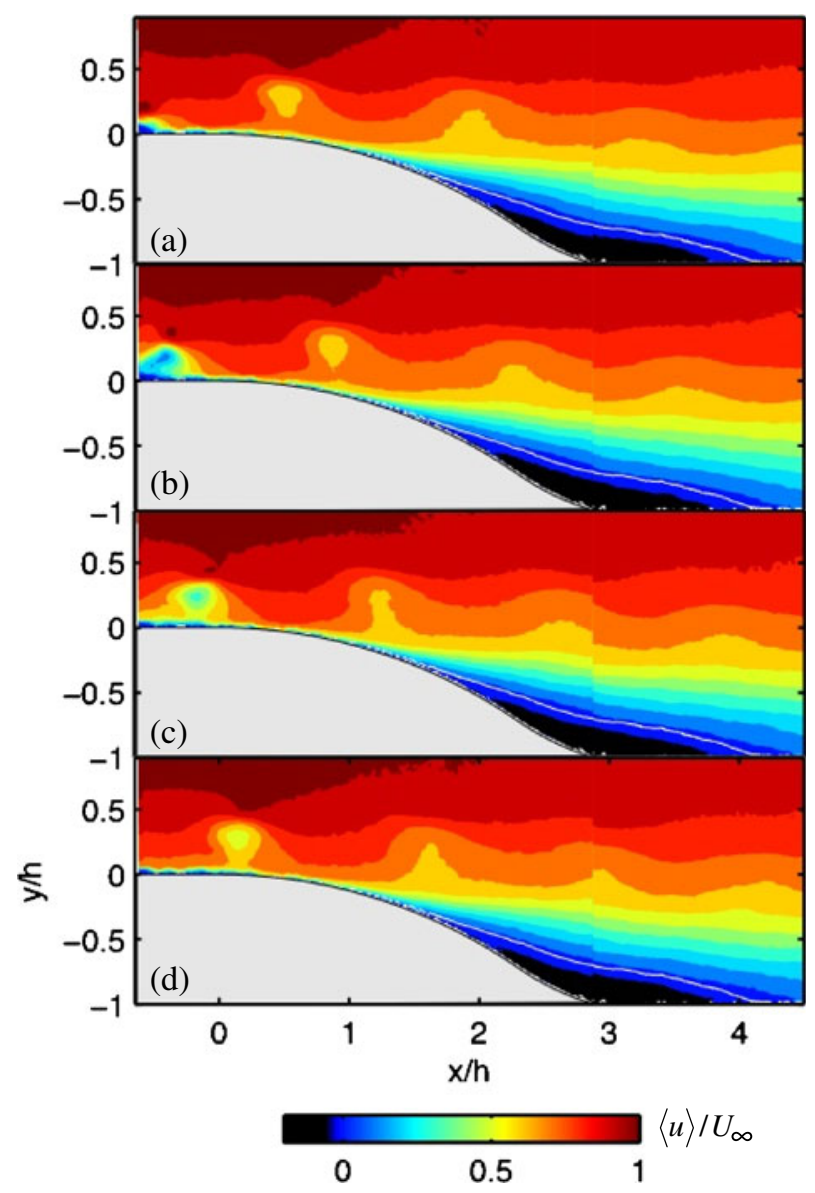


Fig. 6 Contours of phase-averaged spanwise vorticity $\left(\omega_{\mathrm{z}}\right)$ with superimposed velocity vectors at $\mathrm{St}_{\mathrm{h}}=0.6$ and $\mathrm{VR}=0.3$ at four phases in the actuation cycle; $\mathbf{a} 0^{\circ}, \mathbf{b} 90^{\circ}, \mathbf{c} 180^{\circ}$ and d $270^{\circ}$ phase of the actuation cycle

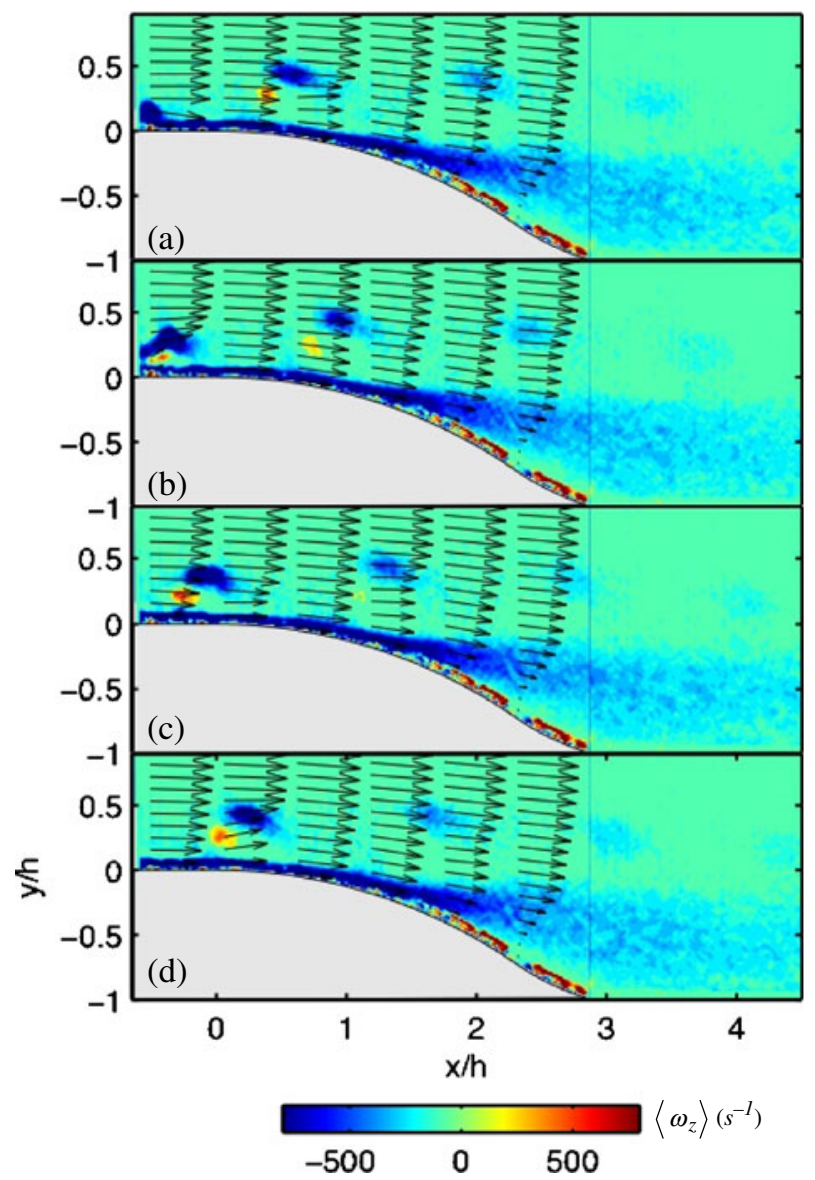

At $\mathrm{St}_{\mathrm{h}}=0.6$, consecutive structures produced by the synthetic jets are more closely spaced and the height of reverse flow remains suppressed throughout the entire actuation cycle (Fig. 5). Although pairs of positive and negative vorticity regions are clearly visible, the region of positive vorticity becomes weaker as the structure propagates downstream and eventually diminishes (Fig. 6). At $\mathrm{St}_{\mathrm{h}}=0.6$, the flapping of shear layer is not evident (Fig. 6).

\subsection{Periodic structures produced by synthetic jets}

Figure 7 shows the contours of periodic streamwise velocity component $\langle\tilde{u}\rangle / U_{\infty}$ at $\mathrm{VR}=0.3$ and $\mathrm{St}_{\mathrm{h}}=0.2$ obtained using the triple decomposition technique. Comparing Fig. 3 with Fig. 7, one can see that although low-speed bulges are the distinct features in the phase-averaged streamwise velocity field, the flow structures produced by the synthetic jets in fact cause alternative regions of positive and negative periodic fluctuations in the boundary layer. The region of negative velocity fluctuations is clearly associated with the low-speed bulges seen in Fig. 3. A further examination of Fig. 7 reveals that both the structures of positive and negative 
Fig. 7 Contours of periodic streamwise velocity at $\mathrm{St}_{\mathrm{h}}=$ 0.2 and $\mathrm{VR}=0.3$ at four phases in the actuation cycle; a $0^{\circ}$, b $90^{\circ}$, c $180^{\circ}$ and $\mathbf{d} 270^{\circ}$. (The red line and the black line are the edge of reverse flow in the baseline and the controlled case respectively)

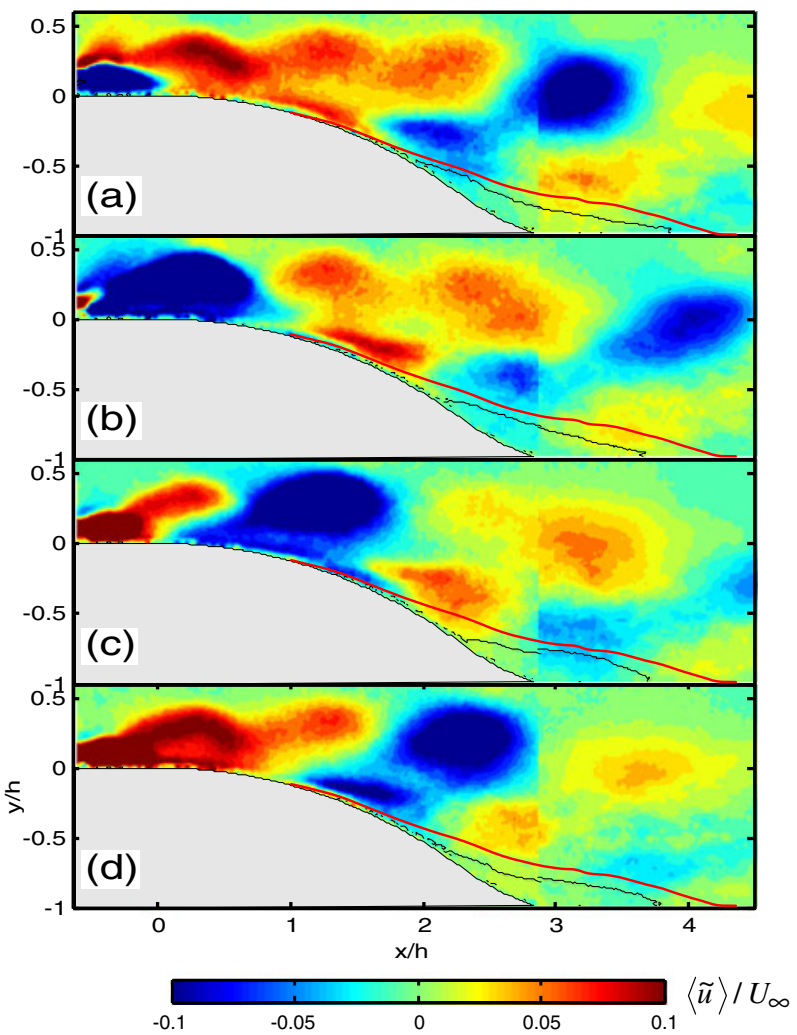

periodic fluctuations appear to be inclined downstream as they move along the ramp and they are also split into two parts height-wise linked with a weaker middle portion. The region of reverse flow is seen to reduce in height when the lower part of the structure associated with the positive periodic fluctuations passes over.

The contours of the periodic vertical velocity component $\langle\tilde{v}\rangle / U_{\infty}$ also reveal the presence of regions of alternative positive and negative periodic fluctuations (see Fig. 8). In comparison with Fig. 7, it is seen that a positive streamwise component is associated with a negative vertical component, especially in the near-wall region. Such a correlation is expected as an upward motion of the fluid in the boundary layer, which lifts up the low-momentum fluid in the near-wall region, will result in a reduction in the local streamwise velocity and verse versa. By correlating Figs. 7 to 8 , one can clearly see that it is the downwards motion of the fluid, which brings the fluid with a higher streamwise velocity to the near-wall region, that leads to a reduction of the height of the separated flow in the controlled case.

At $\mathrm{St}_{\mathrm{h}}=0.6$ and $\mathrm{VR}=0.3$, the regions with alternative positive and negative periodic velocity fluctuations are more closely spaced than at $\mathrm{St}_{\mathrm{h}}=0.2$ as expected (see Fig. 9). Both structures with positive and negative periodic velocity fluctuations appear to become more stretched as they propagate downstream. However, instead of splitting height-wise into two regions of high $\langle\tilde{u}\rangle / U_{\infty}$ as observed at $\mathrm{St}_{\mathrm{h}}=0.2$, there is only one region of high concentration in $\langle\tilde{u}\rangle / U_{\infty}$, which is attached to the wall via a weaker extension. At $\mathrm{St}_{\mathrm{h}}=0.6$, these structures appear shorter in the streamwise 
Fig. 8 Contours of periodic vertical velocity at $\mathrm{St}_{\mathrm{h}}=0.2$ and $\mathrm{VR}=0.3$ at four phases in the actuation cycle; a $0^{\circ}$, b $90^{\circ}$, c $180^{\circ}$ and $\mathbf{d} 270^{\circ}$. (The red line and the black line are the edge of reverse flow in the baseline and the controlled case respectively)

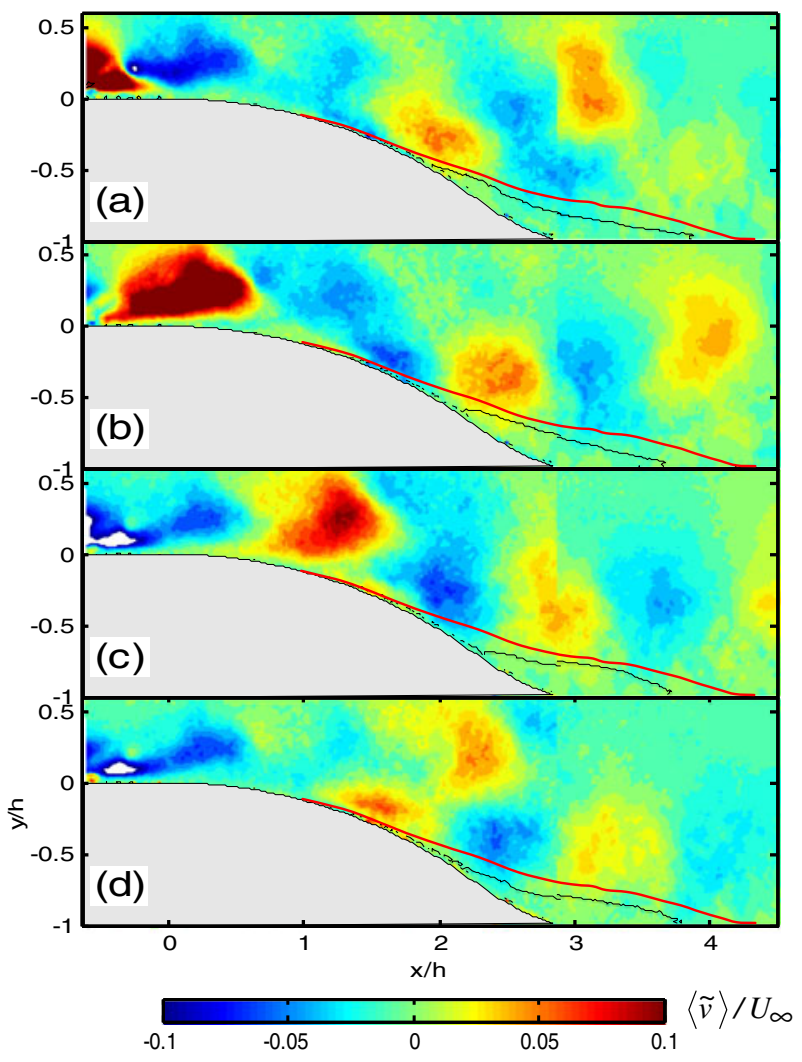

extent and weaker due to a lower $L$, which is required to produce the same VR (see Table 1). It is also worth noting that at $\mathrm{St}_{\mathrm{h}}=0.2$ and $\mathrm{VR}=0.3, L$ is significantly larger than 4 above which secondary vortex rings are shed as the primary vortex becomes saturated $[17,43]$. This results in the formation of larger and more complex vortical structures as seen in Fig. 7.

Furthermore, at $\mathrm{St}_{\mathrm{h}}=0.6$, there are more active structures along a given streamwise length over the ramp. As a result, the streamwise variation in the height of reverse flow becomes less noticeable during one actuation cycle as shown in Fig. 9. Similarly, there is a clear correlation between the regions with positive periodic streamwise velocity fluctuations and those with negative periodic vertical velocity fluctuations (see Fig. 9 and Fig. 10).

\subsection{Effect of synthetic jets on turbulence structures}

With both the PIV and LDA data, the effect of the synthetic jets on the turbulence structures in the separated flow can also be examined in terms of Reynolds stresses, energy flux and power spectra. Here it is of particular interest to see if the turbulence structures are altered in any way to favour the delay of flow separation. 
Fig. 9 Contours of periodic streamwise velocity at $\mathrm{St}_{\mathrm{h}}=$ 0.6 and $\mathrm{VR}=0.3$ at four phases in the actuation cycle; a $0^{\circ}$, b $90^{\circ}$, c $180^{\circ}$ and $\mathbf{d} 270^{\circ}$. (The red line and the black line are the edge of reverse flow in the baseline and the controlled case respectively)

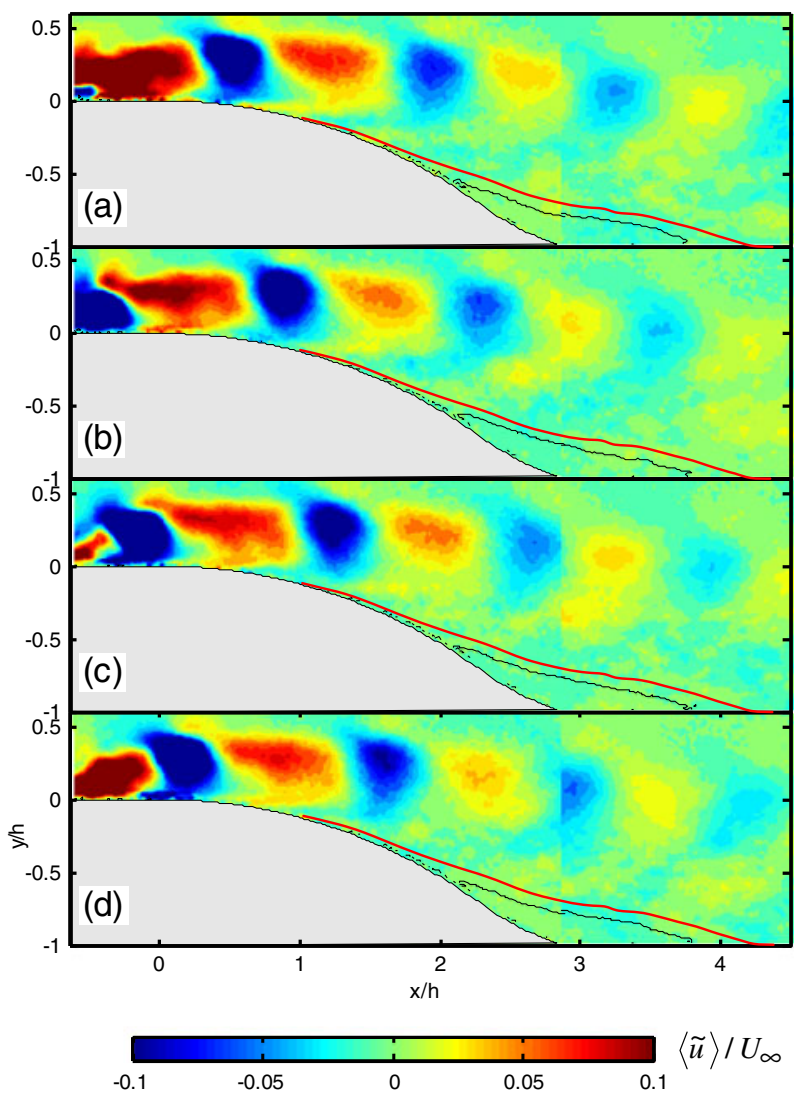

In Figs. 11 and 12 the contours of the Reynolds shear stress $-\left\langle u^{\prime} v^{\prime}\right\rangle / U_{\infty}^{2}$ on the central streamwise plane bisecting the middle jet obtained using $2 \mathrm{D}$ PIV at VR = 0.3 and $\mathrm{St}_{\mathrm{h}}=0.2,0.6$ are shown. The contour lines of positive periodic streamwise velocity fluctuations are superimposed to assist the identification of the response of the separated shear layer to the passage of the structures produced by the synthetic jets. At both $\mathrm{St}_{\mathrm{h}}=0.2$ and 0.6 , the separated shear layer is seen to exhibit a high level of Reynolds shear stress. At $\mathrm{St}_{\mathrm{h}}=0.2$, as the lower portion of the structure of positive periodic streamwise velocity passes through the separated shear layer, the level of $-\left\langle u^{\prime} v^{\prime}\right\rangle / U_{\infty}^{2}$ increases locally and the vertical location of the separated shear layer is deflected downwards correspondingly (see Fig. 11b-d). As shown in Fig. 11a, when a structure of positive periodic streamwise velocity convects over the downstream portion of the reverse flow, the corresponding structure produced in the next cycle has not arrived at the beginning of the separated shear layer. This causes an obvious kink in the separated shear layer. $\mathrm{At} \mathrm{St}_{\mathrm{h}}=0.6$, when the structure of positive periodic streamwise velocity passes, the variation in the intensity and vertical location of the separated shear layer, is less evident (see Fig. 12).

In order to reveal the subtle differences in the response of the separated flow to the actuation of synthetic jets at different phases of a cycle, the phase-averaged 
Fig. 10 Contours of periodic vertical velocity at $\mathrm{St}_{\mathrm{h}}=0.6$ and $\mathrm{VR}=0.3$ at four phases in the actuation cycle; $\mathbf{a} 0^{\circ}, \mathbf{b} 90^{\circ}$, c $180^{\circ}$ and $\mathbf{d} 270^{\circ}$. (The red line and the black line are the edge of reverse flow in the baseline and the controlled case respectively)

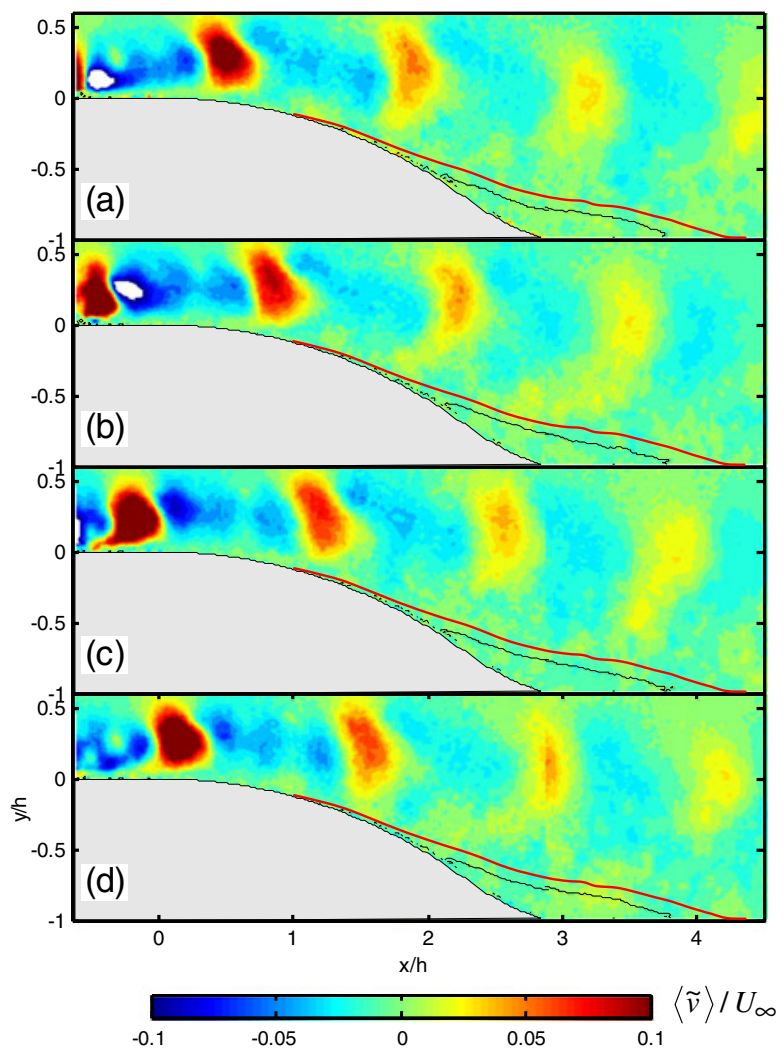

turbulent shear stress distributions across the separated shear layer at $\mathrm{x} / \mathrm{h}=2$ are compared between the baseline case and the controlled cases in Figs. 13 and 14. An increase in the magnitude of turbulence shear stress in the outer part of the separated boundary layer is observed at both actuation frequencies. At $\mathrm{St}_{\mathrm{h}}=0.2$, the lower portion of the structure of positive periodic streamwise velocity arrives at $\mathrm{x} / \mathrm{h}=$ 2 at $90^{\circ}$ phase (see Fig. 11b). This indeed results in a considerable increase in the turbulent shear stress in the separated shear layer at $\mathrm{x} / \mathrm{h}=2$ (Fig. 13b). In contrast, a certain level of decrease is observed at the other phases. Overall, the location of the shear stress spike occupied by the separated shear layer is seen to shift downwards at all phases, being consistent with the reduction of the reverse flow region. However, the downwards shift is more obvious at $90^{\circ}$ and $180^{\circ}$ phase in response to the passage of the lower portion of the structure of positive periodic streamwise velocity. Hence there appears to be a direct connection between the arrival of the regions of positive streamwise velocity fluctuations and the local increase in turbulence level in the separated shear layer at $\mathrm{St}_{\mathrm{h}}=0.2$. At $\mathrm{St}_{\mathrm{h}}=0.6$, the difference in the amount of downwards shift of the shear layer at different phases is less profound. Furthermore, the level of turbulent shear stress remains at a similar level as that in the baseline case (see Fig. 14). 
Fig. 11 Contours of turbulent shear stress $-\left\langle u^{\prime} v^{\prime}\right\rangle / U_{\infty}^{2}$ at $\mathrm{St}_{\mathrm{h}}$ $=0.2$ and $\mathrm{VR}=0.3$ at four phases in the actuation cycle; $\mathbf{a}$ $0^{\circ}$, b $90^{\circ}$, c $180^{\circ}$ and $\mathbf{d} 270^{\circ}$. (The black contour lines represent

$\langle\tilde{u}\rangle / U_{\infty}=0.01$ and 0.05$)$

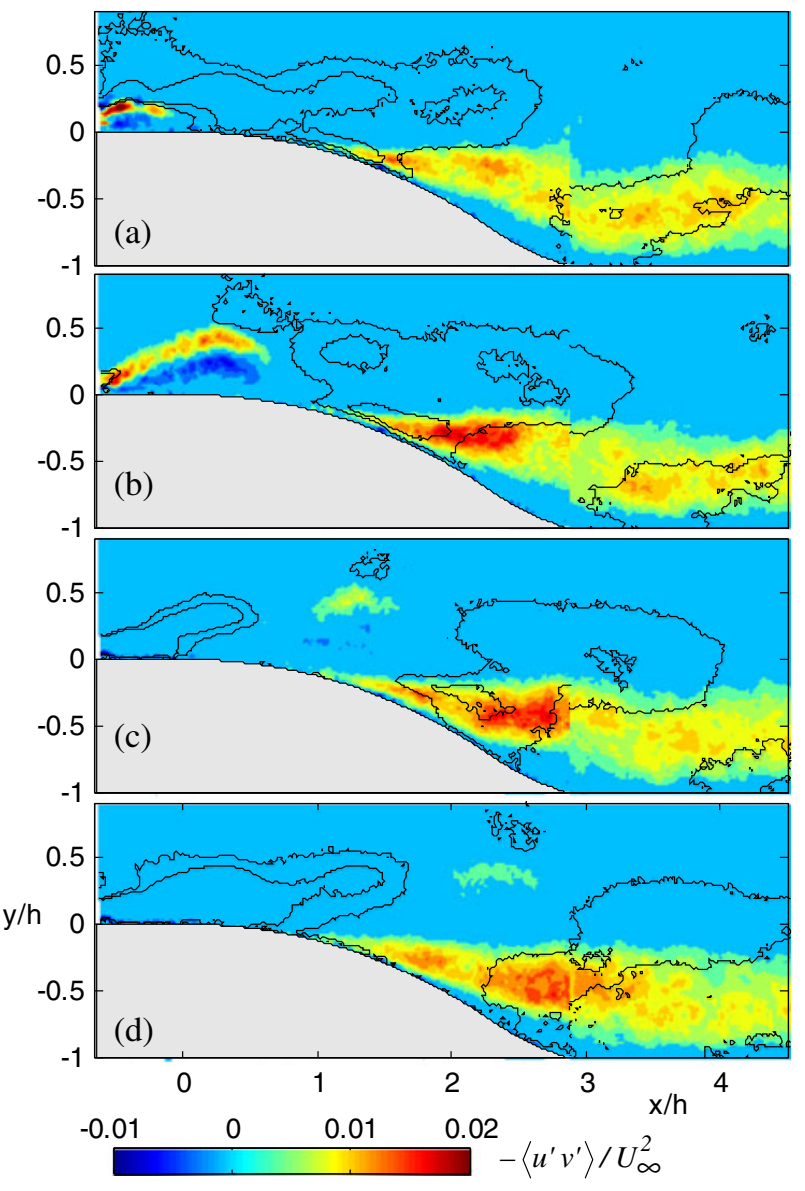

The energy flux associated with the production of random turbulence and periodic structures by extracting energy from the mean flow field can be represented by $-\overline{u^{\prime} v^{\prime}}\left(\frac{\partial \bar{u}}{\partial x}+\frac{\partial \bar{v}}{\partial y}\right)$ and $-\overline{\tilde{u} \tilde{v}}\left(\frac{\partial \bar{v}}{\partial x}+\frac{\partial \bar{u}}{\partial y}\right)$ respectively. In Figs. 15 and 16, the level of energy flux via the formation of random turbulence structures and periodic structures at $\mathrm{St}_{\mathrm{h}}=0.2$ and 0.6 are compared. Figure 15 shows that although the vortical structures produced by the synthetic jets are associated with an appreciable level of energy flux, the turbulence energy flux in the separated shear layer prevails in the controlled cases. Furthermore, the level of energy flux in the separated shear layer is similar at $\mathrm{St}_{\mathrm{h}}=0.2$ and 0.6. In contrast, despite the high level of energy flux associated with the periodic structures in the outer part of the boundary layer, their contribution in the shear layer is significantly less. Nevertheless, at $\mathrm{St}_{\mathrm{h}}=0.2$ there is an appreciable level of periodic energy flux in the shear layer due to an excitation near its natural frequency by the synthetic jets (see Fig. 16a). In contrast, at $\mathrm{St}_{\mathrm{h}}=0.6$ the energy flux produced by the periodic structures in the separated flow region is almost nil (see Fig. 16b). Therefore one can conclude that the flow unsteadiness produced by the 
Fig. 12 Contours of turbulent shear stress $-\left\langle u^{\prime} v^{\prime}\right\rangle / U_{\infty}^{2}$ at $\mathrm{St}_{\mathrm{h}}$ $=0.6$ and $\mathrm{VR}=0.3$ at four phases in the actuation cycle; $\mathbf{a}$ $0^{\circ}$, b $90^{\circ}$, c $180^{\circ}$ and $\mathbf{d} 270^{\circ}$.

(The black contour lines represent

$\langle\tilde{u}\rangle / U_{\infty}=0.01$ and 0.05$)$

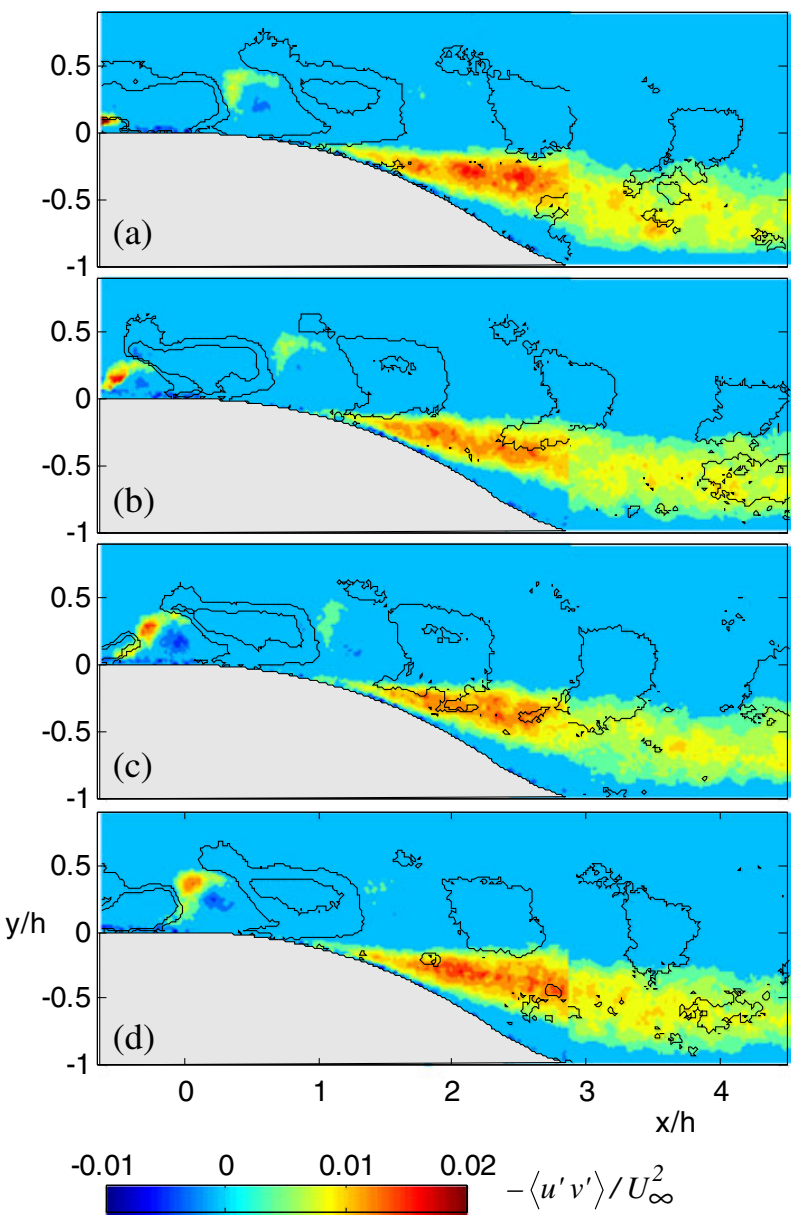

synthetic jets plays a less important role in modifying the separated shear layer at $\mathrm{St}_{\mathrm{h}}=0.6$ than at 0.2 .

Figure 17 indicates that upon the actuation of synthetic jets the energy level at frequencies lower than the natural shear layer frequency in the separated shear layer $(\mathrm{x} / \mathrm{h}=1.8, \mathrm{y} / \mathrm{h}=-0.3)$ is slightly reduced whereas that at higher frequencies is slightly increased, resulting in a visible extension of the inertial subrange to higher frequencies. The effect of synthetic jets at $\mathrm{St}_{\mathrm{h}}=0.2$ and 0.6 appears similar except that a clear peak at the actuation frequency can be identified in the spectrum at $\mathrm{St}_{\mathrm{h}}=0.2$, whereas at $\mathrm{St}_{\mathrm{h}}=0.6$ such a distinct peak is absent. This is consistent with the finding from Fig. 16 that actuating synthetic jets at $\mathrm{St}_{\mathrm{h}}=0.2$ results in an induced oscillation of the shear layer at its natural frequency.

\subsection{Vortical structures responsible for delay of flow separation}

Since stereo PIV measurements produce the three velocity components on crossstream planes, they can provide more information about the characteristics of the 
Fig. 13 Comparison of $-\left\langle u^{\prime} v^{\prime}\right\rangle / U_{\infty}^{2}$ distributions at $\mathrm{x}^{\prime}=2$ between $\mathrm{St}_{\mathrm{h}}=0.2$, $\mathrm{VR}=0.3$ at four phases in the actuation cycle; $\mathbf{a} 0^{\circ}, \mathbf{b} 90^{\circ}$, c $180^{\circ}$ and $\mathbf{d} 270^{\circ}$ and the baseline case. (Black lines: baseline; Blue line: controlled case)

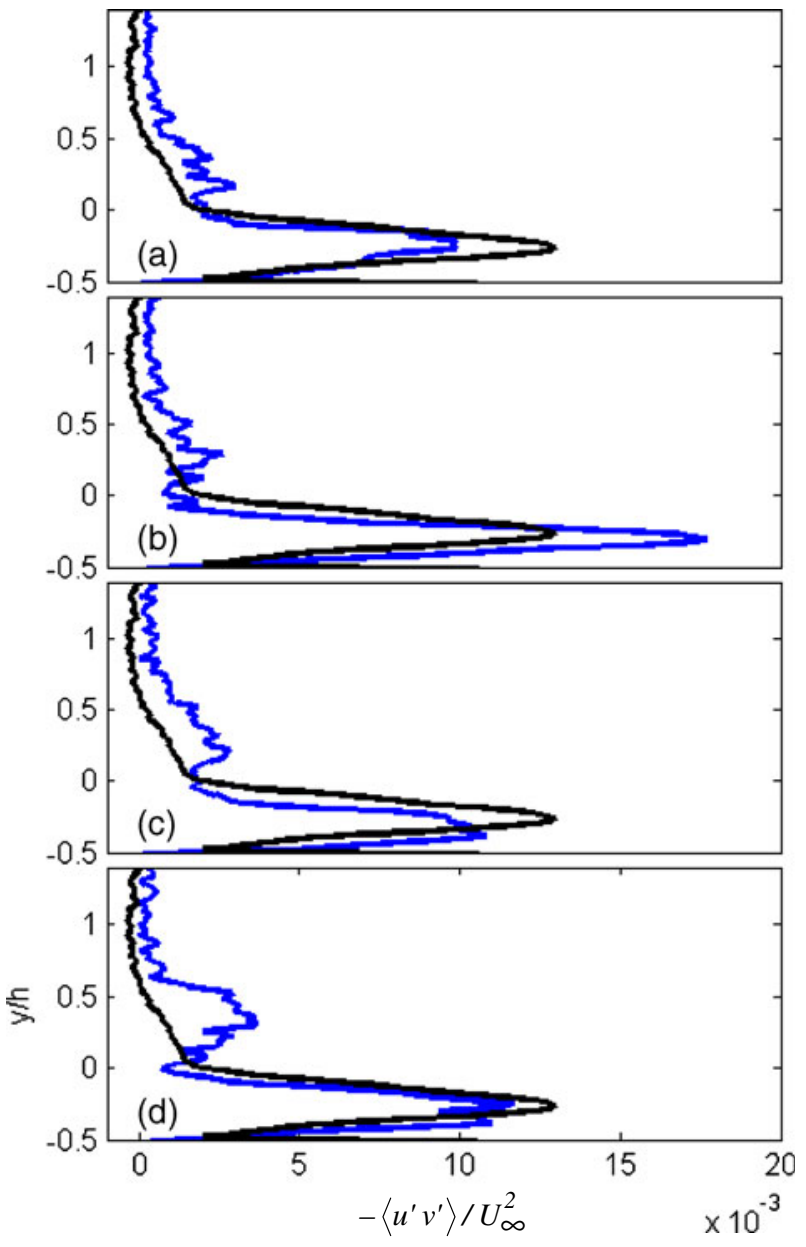

vortical structures produced by the synthetic jet that delay flow separation. In Figs. 18 and 19, the contours of phase-averaged streamwise vorticity and streamwise velocity as these structures pass through the measurement plane at $\mathrm{x} / \mathrm{h}=2$ during an actuation cycle are displayed for $\mathrm{St}_{\mathrm{h}}=0.2$ at $\mathrm{VR}=0.2,0.3$ and 0.5 respectively. The region of reverse flow over the ramp is indicated by the iso-surface of streamwise velocity $\bar{u}=0$ in dark blue and the attached flow appears as the region in the middle, which is void of colour.

At the intermediate velocity ratio of 0.3 , two pairs of streamwise vortices with opposite sense of rotation are revealed by the streamwise vorticity contours with one pair located on the top of the other (see Fig. 18b). The upper pair comes to sight at $\mathrm{t} / \mathrm{T}=0.5$, where $\mathrm{T}$ is the period of synthetic jet actuation, i.e. $\mathrm{T}=1 / \mathrm{f}$. The top vortex pair appears to be inclined downstream since it is located much closer to the wall at a later time. The streamwise vortex pair underneath, which has the opposite sign of rotation, is seen to persist over a substantial portion of the actuation cycle. The maximum control effect occurs around $\mathrm{t} / \mathrm{T}=0.45$ just before the upper 
Fig. 14 Comparison of $-\left\langle u^{\prime} v^{\prime}\right\rangle / U_{\infty}^{2}$ distributions at $\mathrm{x}^{\prime}=2$ between $\mathrm{St}_{\mathrm{h}}=0.6$, $\mathrm{VR}=0.3$ at at four phases in the actuation cycle; $\mathbf{a} 0^{\circ}, \mathbf{b} 90^{\circ}$, c $180^{\circ}$ and $\mathbf{d} 270^{\circ}$ and the baseline case. (Blacklines: baseline; Blueline: controlled case)

Fig. 15 Contours of random turbulent flux $-\overline{u^{\prime} v^{\prime}}\left(\frac{\partial \bar{v}}{\partial x}+\frac{\partial \bar{u}}{\partial y}\right)$ at $\mathrm{VR}=0.3$ and $\mathbf{a} \mathrm{St}_{\mathrm{h}}=0.2$; b $\mathrm{St}_{\mathrm{h}}=0.6$
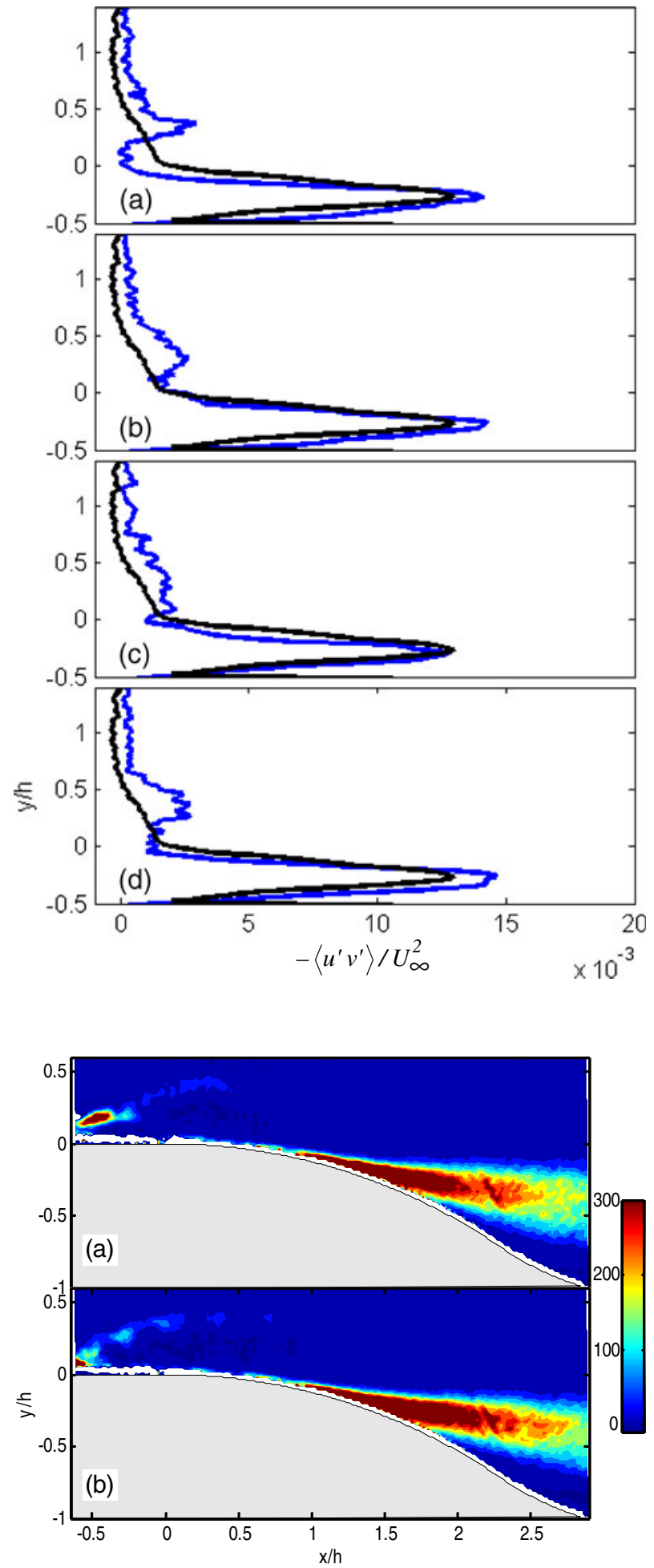
Fig. 16 Contours of periodic energy flux $-\overline{\tilde{u} \tilde{v}}\left(\frac{\partial \bar{v}}{\partial x}+\frac{\partial \bar{u}}{\partial y}\right)$ at $\mathrm{VR}=0.3 \mathbf{a ~ S t} t_{\mathrm{h}}=0.2$ b $\mathrm{St}_{\mathrm{h}}=0.6$

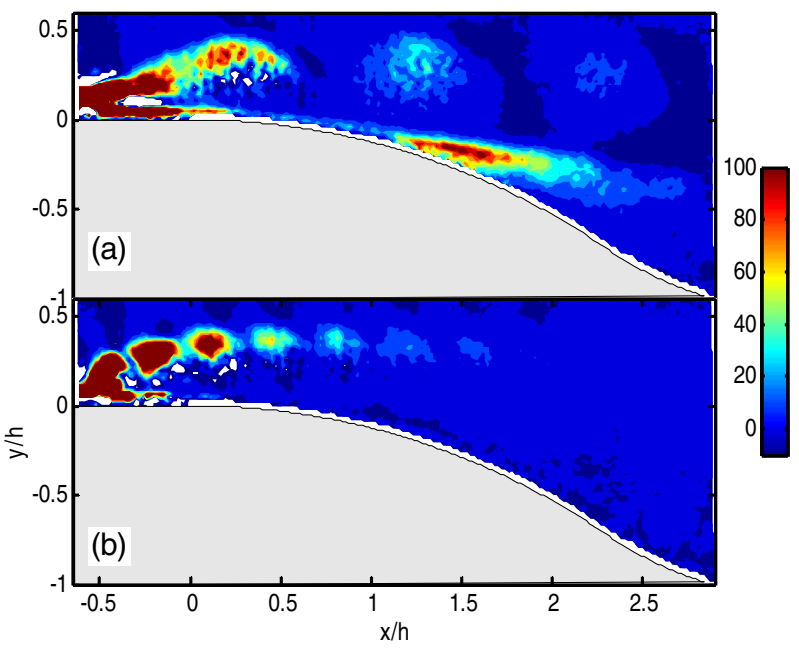

vortex pair arrives, and the spanwise width of the attached flow diminishes towards the end of the cycle as the legs of the upper pair moves nearer to the wall. By correlating the streamwise vorticity and streamwise velocity contours at the same phase (see Figs. 18b and 19b), one can see that the upper vortex pair produces an upwash ejecting low-momentum fluid away from the wall, whereas the lower vortex pair produces a downwash between its limbs bringing high-momentum fluid towards the wall. At the other two velocity ratios, similar structures are observed except that the strength of the vortex pairs is weaker at $\mathrm{VR}=0.2$ and much stronger at $\mathrm{VR}=$ 0.5 (see Fig. 18a and c). The flow control effect increases with the velocity ratio, with both the duration and the maximum width of attached flow being the largest at VR= 0.5. Nevertheless, the flow still remains separated during a portion of the synthetic jet actuation cycle at this measurement location.

Fig. 17 A comparison of power spectra between the baseline and two controlled cases at $\mathrm{VR}=0.3$ in the separated shear layer $(\mathrm{x} / \mathrm{h}=1.8$ and $\mathrm{y} / \mathrm{h}=-0.3)$

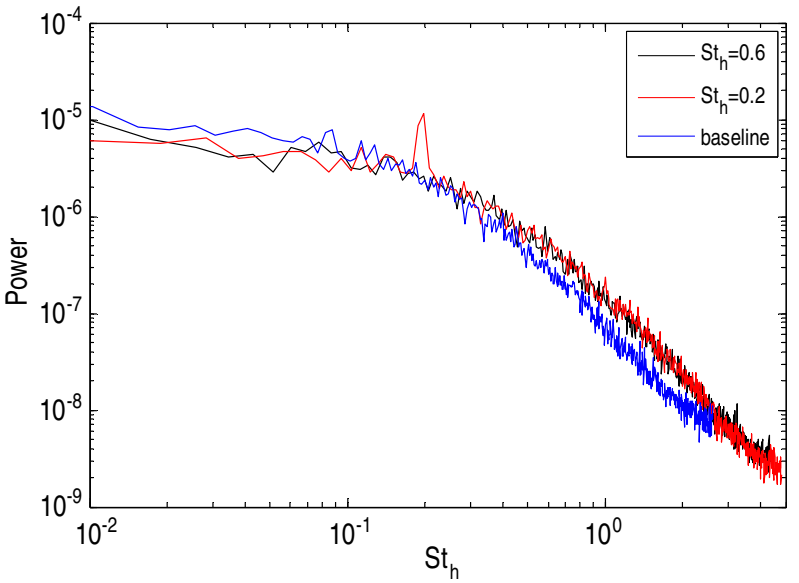


Fig. 18 Contours of phase-averaged streamwise vorticity during a synthetic jet actuation cycle at $\mathrm{x} / \mathrm{h}=2$ for $\mathrm{St}_{\mathrm{h}}=0.2$ at a $\mathrm{VR}=0.2$;

b $\mathrm{VR}=0.3$ and $\mathbf{c} \mathrm{VR}=0.5$

(Blue iso-surface indicates the reverse flow in the separation bubble)

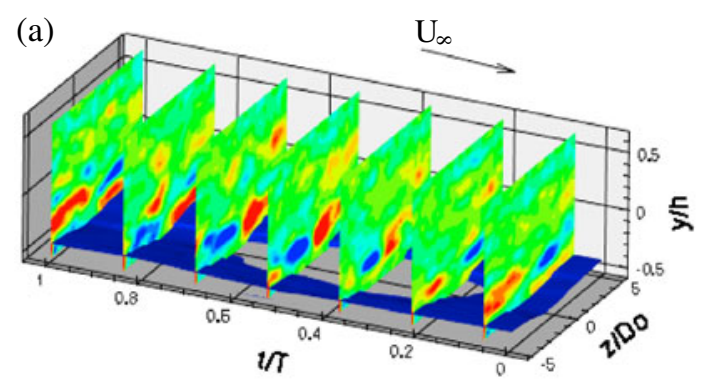

(b)

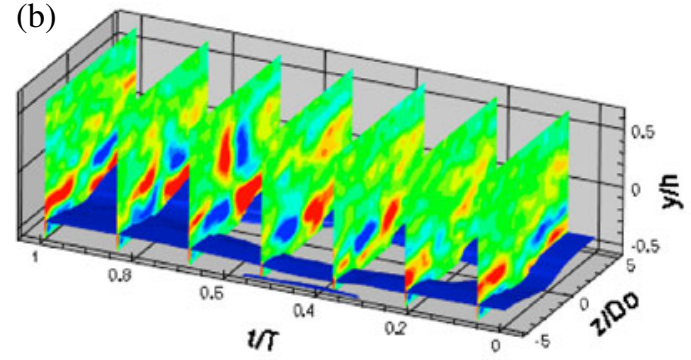

$\left\langle\omega_{x}\right\rangle\left(s^{-1}\right)$

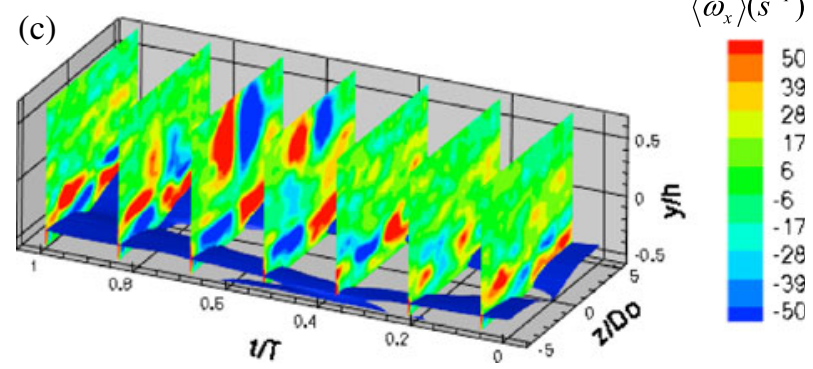

At $\mathrm{St}_{\mathrm{h}}=0.6$, similar vortical patterns and variations in strength with $\mathrm{VR}$ as well as their impact on the streamwise velocity contours are also observed (see Figs. 20 and 21). Nevertheless, compared to $\mathrm{St}_{\mathrm{h}}=0.2$ a much smaller attached flow region is seen at $\mathrm{VR}=0.2$. However, as $\mathrm{VR}$ increases to 0.3 , the flow becomes fully attached over a finite spanwise width during the entire actuation cycle. In addition, in comparison to the case at $\mathrm{St}_{\mathrm{h}}=0.2$, there is much less variation in the local control effect as the synthetic jets pass over.

The physical appearance of the vortical structures observed in Figs. 18 and 20 can be reconstructed from the stereo PIV data using the Q-criterion and the Taylor's frozen structure hypothesis as explained in Section 2.4. Here the convection velocity of the coherent structure is found to be $5.3 \mathrm{~m} / \mathrm{s}$ from the PIV measurement on the streamwise central plane, which is equivalent to $81.5 \%$ of the reference velocity. In Figs. 22 and 23, the iso-surfaces of $\mathrm{Q}=5$ colour-coded with the streamwise vorticity $\omega_{x}$ are shown for $\mathrm{VR}=0.3$ and 0.5 at $\mathrm{St}_{\mathrm{h}}=0.2$ and 0.6 respectively. The value of $\mathrm{Q}$ is chosen by trail-and-error to ensure that the key structures are retained without too many insignificant structures to clutter the appearance. Since the structures at $\mathrm{VR}=0.2$ are too weak and they cannot be seen clearly using this method, the 
Fig. 19 Contours of phase-averaged streamwise velocity during a synthetic jet actuation cycle at $\mathrm{x} / \mathrm{h}=2$ for $\mathrm{St}_{\mathrm{h}}=0.2$ at a $\mathrm{VR}=0.2$;

b $\mathrm{VR}=0.3$ and $\mathbf{c} \mathrm{VR}=0.5$

(Blue iso-surface indicates the reverse flow in the separation bubble)

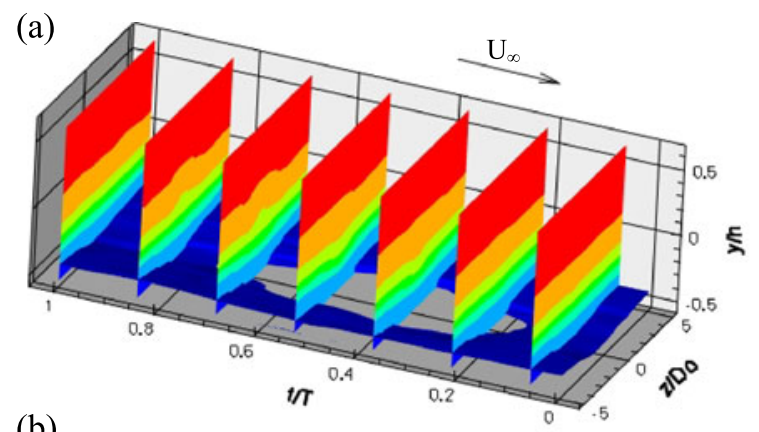

(b)
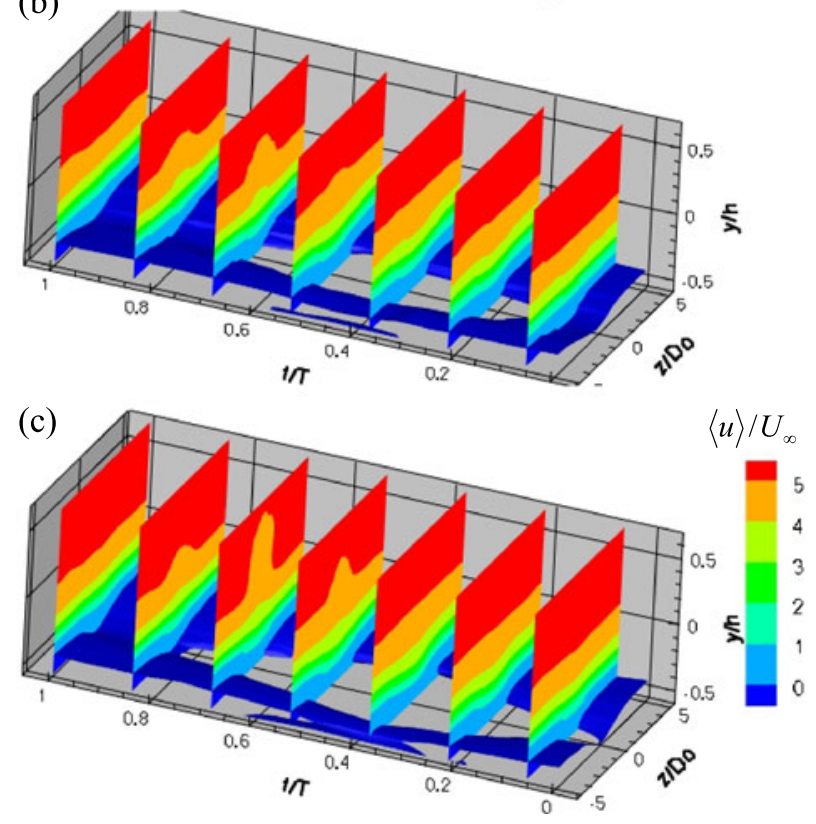

results are omitted here. In the experiment, stereo PIV measurement is made at three streamwise locations $(\mathrm{x} / \mathrm{h}=1,2$ and 2.5$)$ along the ramp. Hence three structures are reconstructed and placed along the ramp to provide a visual impression of how the coherent structure develops as it propagates downstream.

As seen in Figs. 22 and 23, the primary structures produced by the synthetic jets appear as hairpin vortices, which are inclined downstream and located within the local boundary layer. As these hairpin structures convect downstream, they become more stretched and their trailing legs become weaker therefore less visible. Underneath the hairpin vortex, a streamwise vortex pair of the opposite sense of rotation is also visible at the upstream locations. In comparison to the hairpin structure at $\mathrm{St}_{\mathrm{h}}=$ 0.2 , the structure at $\mathrm{St}_{\mathrm{h}}=0.6$ is much weaker and with a shorter streamwise extent. Its legs appear to be more upright whereas its head stretches forward horizontally resulting in a kink where the two parts join together (see Fig. 23b). Based on the findings from the numerical simulations conducted by Zhou and Zhong [44], a hairpin vortex with a shape like this is likely to have evolved from a tilted vortex ring 
Fig. 20 Contours of phase-averaged streamwise vorticity during a synthetic jet actuation cycle at $\mathrm{x} / \mathrm{h}=2$ for $\mathrm{St}_{\mathrm{h}}=0.6$ at a VR $=0.2$;

b $\mathrm{VR}=0.3$ and $\mathbf{c} \mathrm{VR}=0.5$

(Blue iso-surface indicates the reverse flow in the separation bubble)

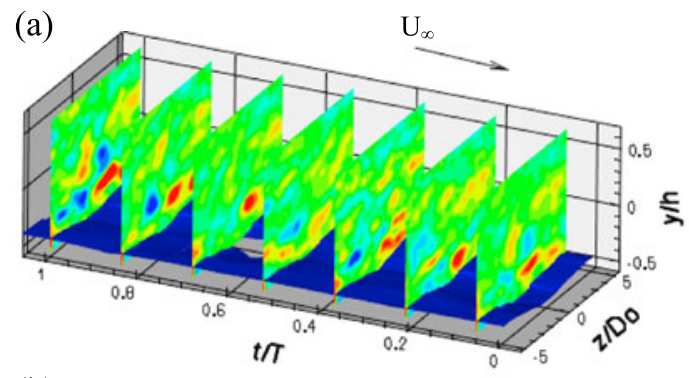

(b)

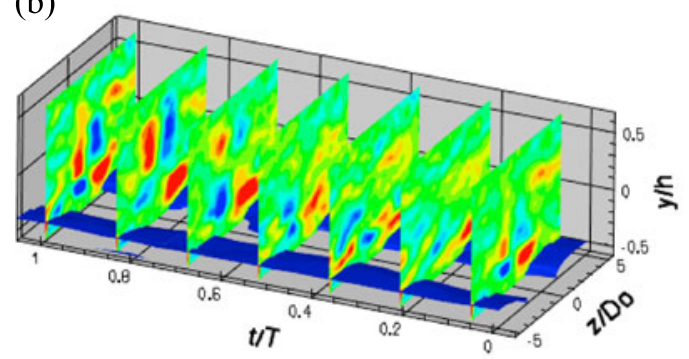

(c)

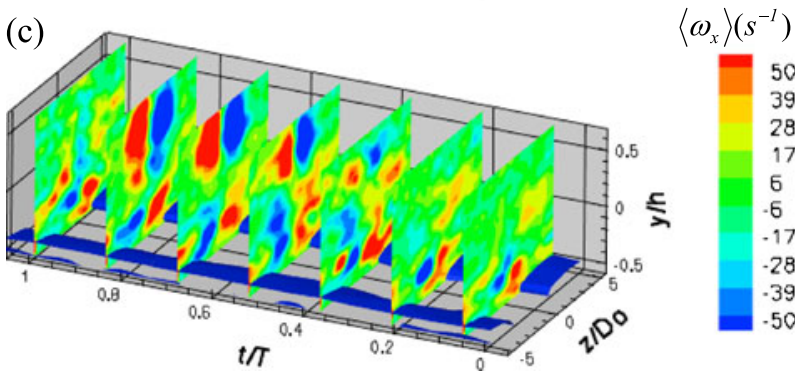

structure, whose upstream portion of the ring has been eventually cancelled out by the resident vorticity in the boundary layer. The diminishing positive vorticity in the pair of positive and negative spanwise vorticity regions as the structures propagate downstream observed in Fig. 6 also provides evidence for support.

\subsection{The mechanism responsible for flow separation delay}

A conceptual model of the vortical structures produced by round synthetic jets, which are responsible for delaying flow separation, has already been put forward by Zhong and Zhong [40]. The analysis of additional experimental data carried out in the present paper is in full support of this model. Indeed, at the test conditions investigated in this study, the primary structures, which are produced by the interaction of synthetic jets and the turbulent boundary layer, are found to be hairpin vortices. These hairpin vortices induce a further pair of streamwise vortices of the opposite sign of rotation underneath themselves. It is the induced streamwise vortices, which are located closer to the wall and persist during a substantial portion of the actuation cycle, that provide the main mechanism of flow separation delay by entraining highmomentum fluids towards the wall. 
Fig. 21 Contours of phase-averaged streamwise velocity during a synthetic jet actuation cycle at $\mathrm{x} / \mathrm{h}=2$ for $\mathrm{St}_{\mathrm{h}}=0.6$ at a $\mathrm{VR}=0.2$;

b $\mathrm{VR}=0.3$ and $\mathbf{c} \mathrm{VR}=0.5$

(Blue iso-surface indicates the reverse flow in the separation bubble)

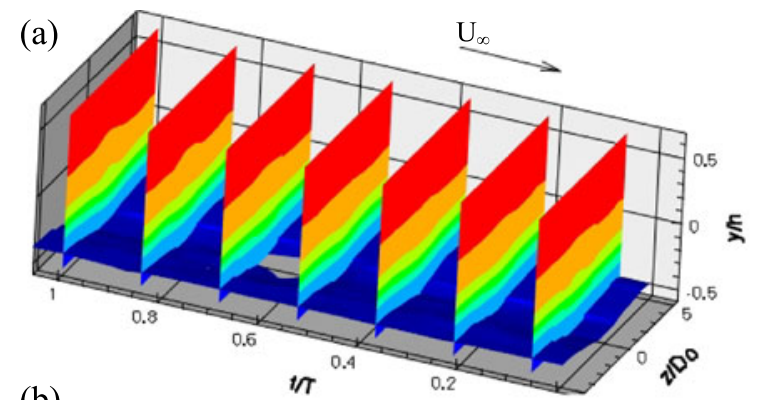

(b)
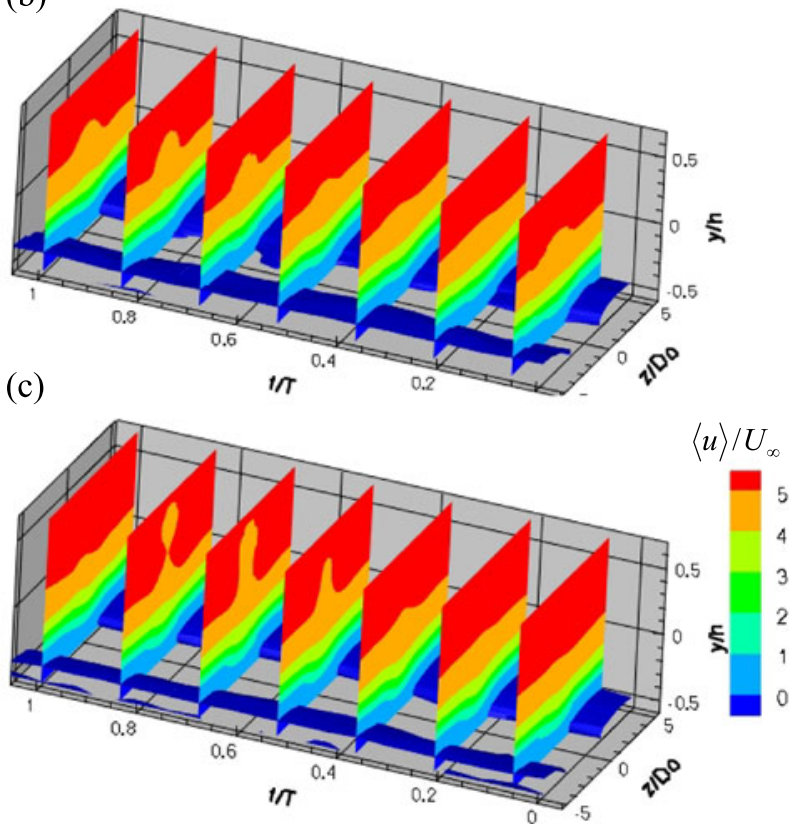

It is noted that a maximum extent of flow separation delay appears just before the hairpin vortex arrives at $\mathrm{St}_{\mathrm{h}}=0.2$ (see Fig. 18) and is accompanied by the arrival of the lower portion of the region with positive periodic fluctuations (see Fig. 7). As shown in the schematic drawing in Fig. 24, the head of the hairpin vortex is located well above the streamwise vortices. Due to its associated spanwise circulation, the head of the hairpin vortex entrains high-momentum fluid downwards in its immediate downstream region. The entrained high-momentum fluid is then brought closer to the wall by the downwards motion produced by the counterrotating streamwise vortex pair located underneath. The two-part region of positive periodic fluctuations observed over the ramp at $\mathrm{St}_{\mathrm{h}}=0.2$ at $\mathrm{VR}=0.3$ (see Fig. 7) is believed to be a manifestation of this chain activity. Here the head of hairpin vortex and the induced streamwise vortex pair appear to work together, providing an effective mechanism of entraining high-momentum fluids from the outer part of the boundary layer to the near-wall region. Such an intense entrainment activity also results in a local increase in turbulence shear stress as seen in Fig. 13b, which favours the delay of flow separation. 
Fig. 22 Iso-surfaces of $\mathrm{Q}$-criterion $(\mathrm{Q}=5)$ colour-coded with streamwise vorticity $\omega_{\mathrm{x}}$ at $\mathrm{St}_{\mathrm{h}}=0.2$ a $\mathrm{VR}=0.3 \mathbf{b} \mathrm{VR}=0.5$
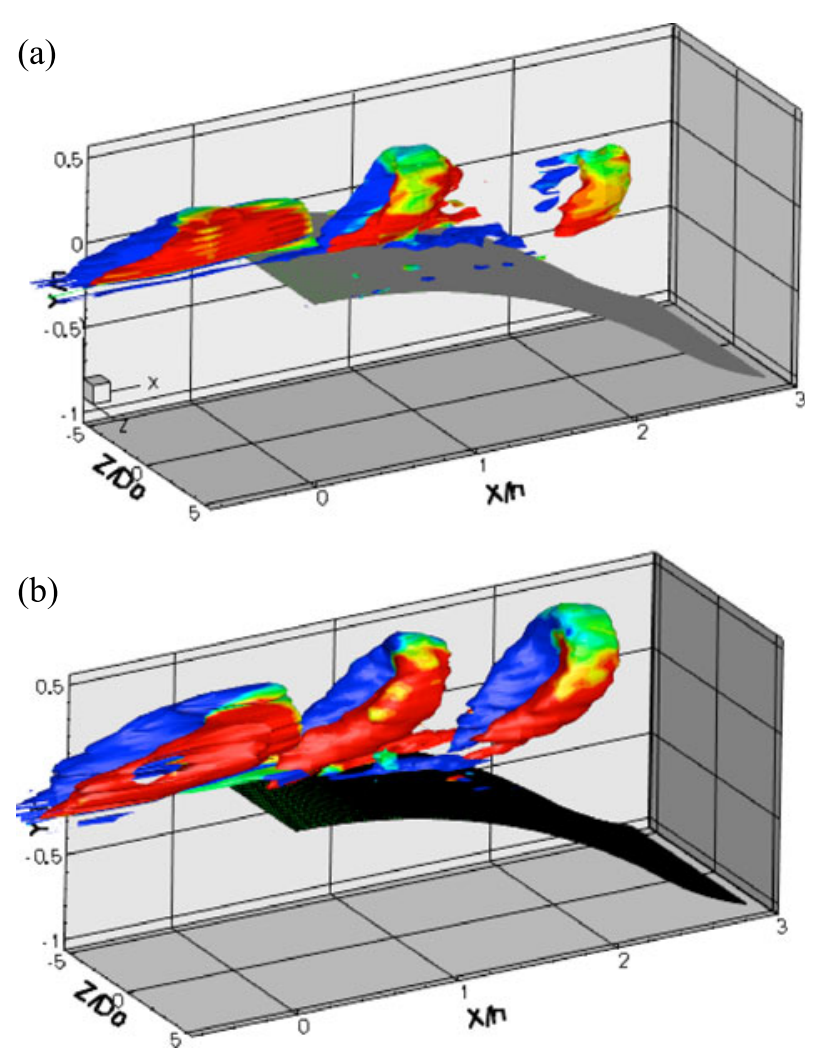

In contrast, the counter-rotating legs of the hairpin vortices are located very close to the induced streamwise vortices. Due to their opposite sense of rotation, a saddle point is formed between these two pairs of vortices (see the insert in Fig. 24). Consequently, the ability of the streamwise vortices in entraining high-momentum fluids towards the wall is reduced because a). The fluid, which is entrained towards the saddle point, is a mixture of the high-momentum fluid from the out part of the boundary layer and the low-momentum fluid nearer to the wall; b). A portion of this entrained fluid is also ejected upwards by the legs of the hairpin vortex. This is likely to be the reason for the reduction of flow control effect at the upstream portion of a hairpin vortex as seen in Fig. 18.

The aforementioned mechanism is expected to remain valid for the cases at $\mathrm{St}_{\mathrm{h}}=0.6$. However, since the vortical structures at $\mathrm{St}_{\mathrm{h}}=0.6$ are more closely packed together and more upright (compare Figs. 22 and 23), the highest level of entrainment tends to occur directly underneath the head of the hairpin vortex rather than further downstream of its head as in the case of $\mathrm{St}_{\mathrm{h}}=0.2$. Together with the weaker nature of these structures as the result of a smaller dimensionless stroke length required for achieving the same VR, the variation in the local control effect as the different portions of the hairpin vortices pass over is therefore dramatically reduced.

The reasons behind the differences in the manner that the extent of time-averaged flow separation delay varies with increasing $\mathrm{VR}$ at $\mathrm{St}_{\mathrm{h}}=0.2$ and 0.6 also become 
Fig. 23 Iso-surfaces of $\mathrm{Q}$-criterion $(\mathrm{Q}=5)$ colour-coded with streamwise vorticity $\omega_{\mathrm{x}}$ at $\mathrm{St}_{\mathrm{h}}=0.6$ a $\mathrm{VR}=0.3 \mathbf{b} \mathrm{VR}=0.5$
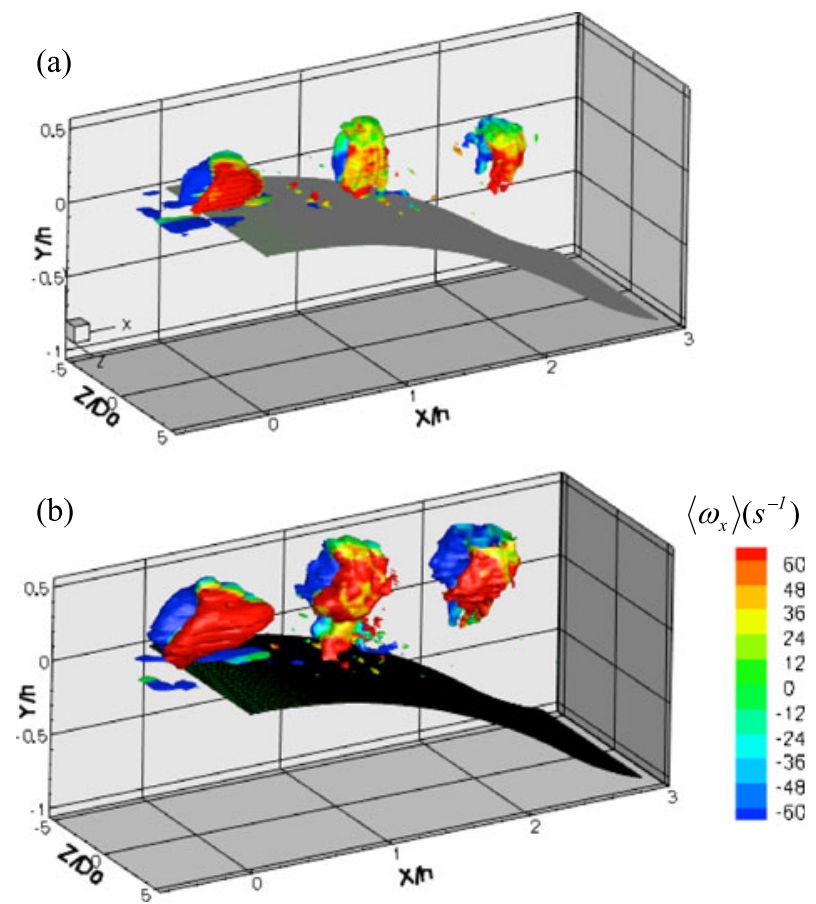

clear. It is seen that at $\mathrm{VR}=0.2$ a much more profound flow control effect is observed at $\mathrm{St}_{\mathrm{h}}=0.2$, however as $\mathrm{VR}$ increases to 0.3 the control effect of $\mathrm{St}_{\mathrm{h}}=0.6$ begins to catch up. At $\mathrm{St}_{\mathrm{h}}=0.6$, the number of active hairpin vortices passing a given location per unit time is increased threefold and as such the separation bubble has less time to regain its original size. It appears that although the vortical structures are comparatively weaker individually than those at $\mathrm{St}_{\mathrm{h}}=0.2$, a further increase in their strength as VR is increased to 0.3 together with closer spaced structures has resulted in a noticeable increase in the flow control effect at $\mathrm{St}_{\mathrm{h}}=0.6$. Hence at $\mathrm{St}_{\mathrm{h}}=0.6$ as VR increases the vortical structures are strengthened further, the flow control effect

Fig. 24 A conceptual model of the vortical structures which produce the delay of flow separation

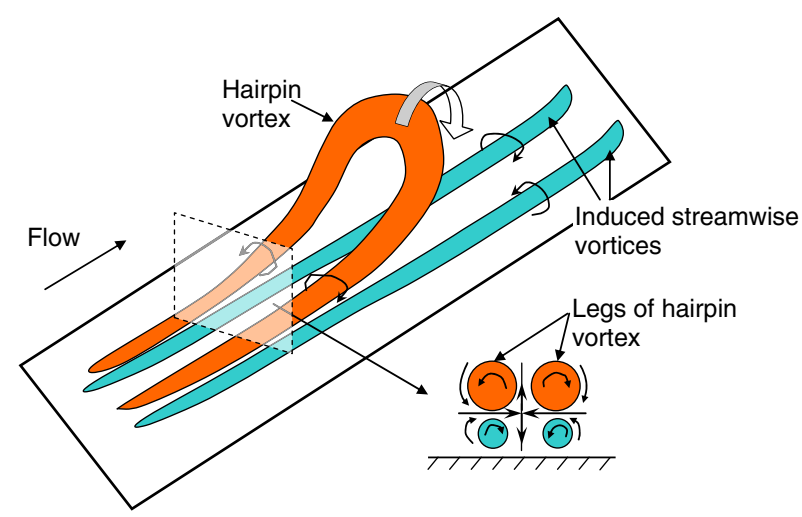


continues to rise steadily. On the other hand, although at $\mathrm{St}_{\mathrm{h}}=0.2$ the structures are capable of suppressing the separation bubble effectively downstream of the head of the hairpin vortex, since flow separation always exists in a certain portion of the actuation cycle (see Fig. 18), the extent of flow separation delay increases more slowly with VR in a time-averaged sense.

In view of the fluctuating behaviour of the reverse flow when the structures produced by synthetic jets pass over the ramp at $\mathrm{St}_{\mathrm{h}}=0.2$, for practical applications on aircraft wings it would be more desirable to operate the jets at $\mathrm{St}_{\mathrm{h}}=0.6 \mathrm{such}$ that the separation is constantly suppressed and the variation in lift is minimized. Nevertheless, it may not be justifiable to assume that the flow control effect will continue to increase with an increasing actuation frequency. According to the numerical simulations results from Zhou [45], who carried out an assessment of the effect of an array of normal circular synthetic jets with a jet spacing of $6 \mathrm{D}_{\mathrm{o}}$ in delaying a laminar flow separation, an optimal actuation frequency exists, which yields consecutive vortical structures with a spacing around the local boundary layer thickness where the synthetic jets are injected. A further increase in the actuation frequency beyond this optimal frequency, however, will result in a deconstructive interaction between consecutive structures hence a decrease in the flow control effect. It is worth noting that in the present experiment, the spacing between consecutive structures at $\mathrm{St}_{\mathrm{h}}=$ 0.6 is only slightly larger than one boundary layer thickness.

The conceptual model described above is expected to be representative of the cases at low to moderate velocity ratios when the jet spacing is relatively large (say not much less than $10 \mathrm{D}_{\mathrm{o}}$ ) and the distance between the jets and the baseline separation line is relatively short. The interaction mechanism may alter when the location of actuators is much further upstream such that the vortical structures have already dissipated by the time they reach the baseline separation or the jet spacing is much smaller such that the interaction between adjacent jets becomes strong. However, this is yet to be verified in future experiments. Furthermore, the present experiment is conducted at a fixed freestream flow velocity and a given pressure gradient. Hence to what extent the findings from this work will change with the level of streamwise pressure gradient is yet to be investigated.

For spanwise slot synthetic jets of large aspect ratios, it is found that when the jets are operated near the shear layer frequency the spanwise vortices produced by the jets will combine with the shedding vortices of the shear layer to form larger spanwise vortical structures, resulting in an enhanced periodic reattachment of the separated flow [11]. In the case of finite-span synthetic jets, the initial 2D spanwise rollers are perturbed by the edge of the slot, leading to formation of multiple distinct streamwise-orientated secondary vortical structures further downstream [29]. In contrast, in the present experiments, round synthetic jets produce streamwise vortical structures immediately downstream of the orifice. Since the head of the hairpins, which exhibits the spanwise vorticity, is well above the separated shear layer, their coupling with the shedding vortices of the shear layer in a separated flow known in the case of slot synthetic jets appears to be absent. Nevertheless, our experimental evidence does show that actuating the synthetic jets near the shear layer frequency causes the separated shear layer to oscillate as the vortical structures produced by the jets pass over (see Figs. 11 and 16a).

It is known that vane vortex generators (VGs) also produce streamwise vortcies [22]. Therefore, there is an overlapping mechanism between synthetic jets and VGs in 
that both of them produce streamwise vortices, which are essential for delaying flow separation. However, the overall vortical structures are different, with a vane vortex generator $(\mathrm{VG})$ producing a single streamwise vortex whereas a round synthetic jet with a low to moderate VR producing a hairpin vortex, which induces a further pair of streamwise vortices underneath. It is shown in this paper that both the hairpin vortices and their streamwise vortices play an active role in enhancing the near-wall mixing.

\section{Conclusion}

In this paper, the 2D and 3D PIV datasets initially presented in Zhang and Zhong [40] are analysed further along with new power spectra deduced from our LDA measurements. In particular, the triple decomposition technique and Q-criterion are employed to investigate how the separated flow responds to the passage of different parts of the vortical structures produced by the synthetic jets during an actuation cycle at different synthetic jet operating conditions. An attempt is made to explain the observed differences in the ways that the separated flow responds to the actuation of synthetic jets at $\mathrm{St}_{\mathrm{h}}=0.2$ and 0.6. A better understanding of the mechanism of flow separation delay using round synthetic jets is obtained, leading to a more complete physical model describing the interaction mechanism than ever attempted before.

It is confirmed that, at the operating conditions tested in the present study, the interaction of the synthetic jets and the turbulent boundary layer results in formation of hairpin vortices, which induce a further pair of streamwise vortices of the opposite sign of rotation underneath. The induced streamwise vortex pair, which are located closer to the wall and persists during a substantial portion of the actuation cycle, is believed to provide the main mechanism of flow separation delay by producing a downwash motion, which brings relatively more energetic fluids towards the wall. Nevertheless, the heads of the hairpin vortices located above also play an important role by supplying high-momentum fluids from the outer portion to the inner portion of the boundary layer, which is subsequently brought to the near-wall region by the induced streamwise vortices. This is manifested as a stronger local flow control effect downstream and underneath their heads, which is particularly obvious at $\mathrm{St}_{\mathrm{h}}=0.2$.

The observed differences in the ways that the separated flow responds to the actuation of synthetic jets at $\mathrm{St}_{\mathrm{h}}=0.2$ and 0.6 are believed to be caused by the differences in the size, strength, and spacing of the hairpin structures produced by the synthetic jets at different actuation frequencies. At the same VR, the structures produced at $\mathrm{St}_{\mathrm{h}}=0.2$ are stronger and have a larger streamwise extent hence they are capable of producing a more profound local flow control effect. However, since they are more spatially apart the height of the reverse flow region is found to experience a rebound after the hairpin structure produced by the synthetic jet has passed and the reverse flow is only partially eliminated during an actuation cycle. In contrast, since at $\mathrm{St}_{\mathrm{h}}=0.6$ the number of active hairpin vortices passing over a given location per unit time is increased threefold, the reverse flow has less time to regain its original size. Hence when the vortical structures have gained a sufficient strength as VR increases to 0.3 and above, the reverse flow can be inhibited during the entire actuation cycle despite their comparatively weaker individual strength. Furthermore, although an actuation of synthetic jets at $\mathrm{St}_{\mathrm{h}}=0.2$ and 0.6 both results in a slight 
shift of turbulence energy to higher frequencies, the separated shear layer exhibits an unsteady oscillation at $\mathrm{St}_{\mathrm{h}}=0.2$ due to its closeness to the natural frequency of the separated shear layer.

Some useful guidelines on flow separation control in practical settings, such as on aircraft wings, can also be derived from this work. In view of the oscillatory response of the separated flow to the actuation of synthetic near the shear layer frequency, it would be desirable to operate the jets at a higher frequency (for example $\mathrm{St}_{\mathrm{h}}=$ $0.6)$ such that the separation is constantly suppressed and the variation in the lift is minimized.

Acknowledgements The work presented in this paper was sponsored by the Engineering and Physical Sciences Research Council in the UK (grant reference EP/E047041/1) and the Airbus UK. It was conducted as part of a joint research programme with Imperial College London where LES simulations of the same test geometry were undertaken. The authors would like to thank the research team at Imperial under the direction of Professor Michael Leschziner for many inspirational discussions. The valuable comments from the industrial coordinator at Airbus, Dr Norman Wood, are also gratefully acknowledged.

\section{References}

1. Amitay, M., Cannelle, F.: Evolution of finite-span synthetic jets. Phys. Fluids 18, 054101 (2006)

2. Amitay, M., Smith, D.R., Kibens, V., Parekh, D.E., Glezer, A.: Aerodynamic flow control over an unconventional airfoil using synthetic jet actuators. AIAA J. 39(3), 361-370 (2001)

3. Bentaleb, Y., Lardeau, S., Leschziner, M.A.: Large-eddy simulation of turbulent boundary layer separation from a rounded step. J. Turbul. 13(6), 1-28 (2012)

4. Cater, J.E., Soria, J.: The evolution of round zero-net-mass-flux jets. J. Fluid Mech. 472, 167-200 (2002)

5. Cattafesta, L.N., Sheplak, M.: Actuators for active flow control. Ann. Rev. Fluid Mech. 43, 247$72(2011)$

6. Clancy, L.J.: Aerodynamics. Pitman Publishing Limited, London (1975)

7. Crook, A., Sadri, A.M., Wood, N.J.: The development and implementation of synthetic jets for the control of separated flow. In: The 17th AIAA Applied Aerodynamics Conference, AIAA paper 99-3176, Norfolk, VA, USA (1999)

8. Dandois, J. Garnier, E., Sagaut, P.: Numerical simulation of active separation control by a synthetic jet. J. Fluid Mech. 574, 25-58 (2007)

9. Gad-El-Hak, M.: Flow control: passive, active and reactive flow management, 1st edn. Cambridge University Press (2000)

10. Garcillian, L, Liddle, S, Zhong, S., Wood, N.J.: Time evolution of the interaction of synthetic jets with a turbulent boundary layer. CEAS/KATnet Conference on Key Aerodynamic Technologies, Bremen, Germany (2005)

11. Greenblatt, D., Paschal, K.B., Yao, C-S., Harris, J.: Experimental investigation of separation control. Part 2: zero mass-flux oscillatory blowing. AIAA J. 44(12), 2831-2845 (2006)

12. Glezer, A., Amitay, M.: Synthetic jets. Ann. Rev. Fluid Mech. 34, 503-529 (2002)

13. Hasan, M., Khan, A.: On the instability characteristics of a reattaching shear layer with nonlaminar separation. Int. J. Heat Fluid Flow 13, 224-23 (1992)

14. Holman, R., Utturkar, Y., Mittal, R., Smith, B.L., Cattafesta, L.: Formation criterion for synthetic jets. AIAA J. 43(10), 2110-2116 (2005)

15. Hunt, J.C.R., Wray, A.A., Moin, P.: Eddies, stream, and convergence zones in turbulent flows. Center for Turbulence Research, Report CTR-S88, Stanford University (1988)

16. Iai, T., Motosuke, M., Honami: Vortex behavior of vertical and inclined synthetic jet in cross flow at low Reynolds number. AIAA Paper 2009-4178 (2009)

17. Jabbal, M., Wu, J., Zhong, S.: The performance of round synthetic jets in quiescent flow. Aeronaut. J. 110(1108), 385-393 (2006)

18. Jabbal, M., Zhong, S.: The near-wall effect of synthetic jets in a boundary layer. Int. J. Heat Fluid Flow 29(1), 119-130 (2008) 
19. Jabbal, M., Zhong, S.: Particle image velocimetry measurements of the interaction of synthetic jets with a zero-pressure gradient laminar boundary layer. Phys. Fluids 22, 063603 (2010)

20. Kotapati, R.B., Mittal, R., Marxen, O., Ham, F., You, D., Cattafesta, L.N.: Nonlinear dynamics and synthetic-jet-based control of a canonical separated flow. J. Fluid Mech. 654, 65-97 (2010)

21. Leschziner, M.A., Lardeau, S.: Simulation of slot and round synthetic jets in the context of boundary-layer-separation control. Philos. Trans. R. Soc. A Math. Phys. Eng. Sci. 370(1), 1 (2010)

22. Lin, C.J.: Review of research on low-profile vortex generators to control boundary-layer separation. Prog. Aerosp. Sci. 38, 389-420 (2002)

23. Lockerby, D.A., Carpenter, P.W.: Modeling and design of microjet actuators. AIAA J. 42(2), 220-227 (2004)

24. Milanovic, I.M., Zaman, K.B.M.Q.: Synthetic jets in cross flow. AIAA J. 43(7), 929-940 (2005)

25. McCormick, D.C.: Boundary layer separation control with directed synthetic jets. AIAA Paper 2000-0519 (2000)

26. Nobach, H.: A global concept of autocorrelation and power spectral density estimation from LDA data sets. In: The 10th International Symposium on Applications of Laser Techniques to Fluid Mechanics, Lisbon, Portugal (2000)

27. Prasad, A.K.: Stereoscopic particle image velocimetry. Exp. Fluids 29, 130-116 (2000)

28. Reynolds W.C., Hussain, A.K.M.F.: The mechanics of an organized wave in turbulent shear flow. J. Fluid Mech. 54, 263-288 (1997)

29. Sahni, O., Wood, J., Jansen, K., Amitay, A.: Three-dimensional interactions between a finitespan synthetic jet and a cross-flow. J. Fluid Mech. 671, 254-287 (2011)

30. Sau, R., Mahesh, K.: Dynamics and mixing of vortex rings in crossflow. J. Fluid Mech. 604, 389409 (2008)

31. Sauerwein, S.C., Vakili, A.D.: An experimental study of zero-mass jets in crossflow. AIAA Paper 99-0068 (1999)

32. Song, S., Eaton, J.K.: Reynolds number effects on a turbulent boundary layer with separation, reattachment, and recovery. Exp. Fluids 36(2), 246 (2004)

33. Tang, H., Zhong, S., Jabbal, M., Garcillan, L., Guo, F., Wood, N.J., Warsop, C.: Towards the design of synthetic-jet actuators for full-scale flight conditions. Part 2: Low-dimensional actuator prediction models and actuator design methods. Flow Turbul. Combust. 78(3), 309-329 (2007)

34. Tennekes, H., Lumley, J.L.: A first course in turbulence. MIT Press (1972)

35. Taylor, G.I.: The spectrum of turbulence. Proc. R. Soc. Lond. A 164, 476 (1938)

36. Wu, D.K., Leschziner, M.A.: Large-eddy simulations of circular synthetic jets in quiescent surroundings and in turbulent cross-flow. Int. J. Heat Fluid Flow 30(3), 421-434 (2009)

37. Zaman, K.B., Milanovic, I.M.: Synthetic jets in cross-flow: part1- round jet. AIAA Paper 20033714 (2003)

38. Zang, W., Prasad, A.K.: Performance evaluation of a Scheimpflug stereoscopic particle image velocimetry. App. Opt. 36, 8738-8744 (1997)

39. Zhang, S., Zhong, S.: An experimental investigation of laminar flow separation control using an array of synthetic jets. AIAA J. 48(3), 611-623 (2010)

40. Zhang, S., Zhong, S.: Turbulent flow separation over a two-dimensional ramp using synthetic jets. AIAA J. 49(12), 2637-2649 (2011)

41. Zhong, S., Jabbal, M., Tang, H., Garcillan, L., Guo, F., Wood, N.J., Warsop, C.: Towards the design of synthetic-jet actuators for full-scale flight conditions. Part 1: The fluid mechanics of synthetic-jet actuators. Flow Turbul. Combust. 78(3), 283-307 (2007)

42. Zhong, S., Millet, F., Wood, N.J.: The behaviour of synthetic jets in a laminar boundary layer. Aeronaut. J. 109(1100), 461-470 (2005)

43. Zhou, J., Zhong, S.: Numerical simulations of the interaction of circular synthetic jets with a laminar boundary layer. Comput. Fluids 38, 393-405 (2009)

44. Zhou, J., Zhong, S.: Coherent structures produced by the interaction between synthetic jets and a laminar boundary layer and their surface shear stress patterns. Comput. Fluids 39, 1296-1313 (2010)

45. Zhou, J.: Numerical simulations of circular synthetic jets for flow separation control. Ph.D. Dissertation, School of Mechanical, Aerospace and Civil Engineering, Manchester University (2010) 